\title{
Numerical Study of Hierarchical Quantum Hall Edge States on the Disk Geometry
}

\author{
Andrea Cappelli \\ I.N.F.N. and Dipartimento di Fisica, Largo E. Fermi 2, I-50125 Firenze, Italy \\ Carlos Méndez \\ Departamento de Física, Pontifícia Universidade Católica, C.P. 38071, 22452-970 Rio de Janeiro,RJ, Brazil \\ Jorge M. Simonin \\ Centro Atómico Bariloche, Comisión Nacional de Energía Atómica and Instituto Balseiro, Universidad Nacional de Cuyo \\ 8400 - San Carlos de Bariloche Río Negro, Argentina \\ Guillermo R. Zemba \\ Centro Atómico Bariloche, Comisión Nacional de Energía Atómica and Instituto Balseiro, Universidad Nacional de Cuyo \\ 8400 - San Carlos de Bariloche Río Negro, Argentina
}

\begin{abstract}
We present a detailed analysis of the exact numerical spectrum of up to ten interacting electrons in the first Landau level on the disk geometry. We study the edge excitations of the hierarchical plateaus and check the predictions of two relevant conformal field theories: the multi-component Abelian theory and the $W_{1+\infty}$ minimal theory of the incompressible fluids. We introduce two new criteria for identifying the edge excitations within the low-lying states: the plot of their density profiles and the study of their overlaps with the Jain wave functions in a meaningful basis. We find that the exact bulk and edge excitations are very well reproduced by the Jain states; these, in turn, can be described by the multi-component Abelian conformal theory. Most notably, we observe that the edge excitations form sub-families of the low-lying states with a definite pattern, which is explained by the $W_{1+\infty}$ minimal conformal theory. Actually, the two conformal theories are related by a projection mechanism whose effects are observed in the spectrum. Therefore, the edge excitations of the hierarchical Hall states are consistently described by the $W_{1+\infty}$ minimal theory, within the finite-size limitations.
\end{abstract}

\section{INTRODUCTION}

One of the important open problems in the physics of the quantum Hall effect (QHE) [1] [2] is the complete understanding of the hierarchical Hall plateaus, whose filling fractions fall beyond the Laughlin sequence $\nu=1,1 / 3,1 / 5, \ldots$ [3]. There are two kinds of theoretical descriptions available at present: the wave-function constructions and the effective conformal field theories (CFT) in $(1+1)$ dimensions.

The first approach has culminated in the Jain theory of the composite-fermion correspondence between the integer Hall states with $\nu^{*}=m=2,3, \ldots$ and the hierarchical states with $\nu=m /(m p \pm 1), p=2,4, \ldots$, such as $\nu=$ $2 / 5,3 / 7, \ldots$ 活]. The existence of the composite-fermion excitations has been confirmed by many experiments [5]; the corresponding ansatz wave-functions have been tested in numerical simulations of few electron systems [- [4] [8]. These have been mostly done on the spatial geometry of the sphere and have firmly established that the Jain states describe the bulk excitations of quantum Hall fluids. On the other hand, these incompressible fluids have characteristic edge excitations [9], which cannot be seen on the sphere geometry. These excitations are the relevant low-energy degrees of freedom in the conduction experiments, and their basic properties, like the fractional charge, have been already measured in the simplest (yet non-trivial) case of the $\nu=1 / 3$ Laughlin Hall state [10] [11 [12.

The edge excitations are naturally described by the conformal field theories] 14, because their low-energy dynamics is effectively one-dimensional, being localized on the boundary of the sample within a width of the order of the magnetic length $\ell=\hbar c / e B \sqrt{15}[16]$. The conformal field theories are a powerful tool because they can be solved explicitly in a non-perturbative framework [14], and predict universal data like the filling fraction, the fractional charge and

\footnotetext{
${ }^{1}$ Moreover, the mean-field theory of the composite fermion has been developed in Ref. [6].

${ }^{2}$ An equivalent language is given by the topological Chern-Simons theories in $(2+1)$ dimensions [13].
} 
quantum statistics of the edge excitations; moreover, they directly describe the relevant experimental regime of a large number of electrons.

However, these effective descriptions cannot be easily related to the microscopic dynamics of the electrons. Actually, based on simple arguments and symmetry principles, two classes of CFTs have been proposed for the edge excitations of the hierarchical plateaus. The first is given by the $m$-component Abelian theories $\widehat{U(1)}{ }^{m}$, which are generalizations of the successful one-component theory for the Laughlin plateaus [17] [13] [9]. In the literature, these conformal theories have been naturally associated with the Jain approach, because the composite-fermion correspondence implies the existence of several effective Landau levels, which may have independent edges of the Laughlin type.

The second class of conformal field theories encompasses the $W_{1+\infty}$ minimal models [18], which exploit the $W_{1+\infty}$ symmetry of the incompressible fluids under area-preserving diffeomorphisms of the plane [19] [20] [21]. There is a one-to-one correspondence between the hierarchical Hall plateaus and the $W_{1+\infty}$ minimal theories. These theories can be obtained by projecting out some states of the multi-component Abelian theory, those which are not fluctuations of an elementary incompressible fluid. This projection implies different properties for the edge excitations in the two theories, which are qualitatively and quantitatively important [18.

In this paper, we study numerically the spectrum of a finite systems of $N=6,8$ and 10 electrons on a disk geometry; we diagonalize exactly the Hamiltonian with the Haldane short-range interaction in the first Landau level [22]. The low-lying states occur in branches which are separated by gaps; each branch contains one state of lowest angular momentum, the "bottom" state, followed by several higher angular momentum levels with close energies (see Fig.(11)). This pattern is well understood for the $\nu=1 / 3$ Laughlin Hall fluid: the bottom state is the ground state, which is an exact eigenstate of the Haldane interaction; the higher levels are the degenerate edge excitations. The plot of density profiles shows that the ground state is rather flat, a characteristic of incompressible fluids [3], and that the edge excitations are infinitesimal density deformations.

We use the density plots to gain a qualitative understanding of the spectrum: we find that the other bottom states can be either quasi-particle excitations (oscillating density profile), or new incompressible fluids with lower filling fractions, such as $\nu=2 / 5$ (flat density profile).

Furthermore, we overlap the low-lying states with the Jain ansatz states. The Jain composite-fermion theory on the disk geometry does predict the branches in the spectrum: they correspond to effective Landau levels which are filled selectively at the boundary. These states are usually denoted by $\left[n_{1}, n_{2}, \ldots, n_{k}\right]$, and correspond to filling the $i$-th level with $n_{i}$ electrons, with $n_{1} \geq n_{2} \geq \cdots \geq n_{k}$ and $\sum n_{i}=N$ 俚. Our numerical analysis shows that each branch in the spectrum is well described by one of these states, corresponding to a given set of fillings $\left\{n_{i}\right\}$, and by the corresponding particle-hole excitations.

We then find the following results. Smooth density profiles identify unambiguously the branches corresponding to the $\nu=2 / 5$ and $3 / 7$ hierarchical plateaus, for each value of $N$; the rest are quasi-particles branches over the $\nu=1 / 3$ Laughlin and the hierarchical plateaus; previous numerical analyses [9] [23] 24] only charted the energy spectrum and were naturally led to misinterpret the branches.

Next, we observe that, within each branch, the low-lying states can be divided into families of close density profiles: the edge excitations correspond to infinitesimal deformations of the bottom state, while families with different density profiles are other bulk excitations or magneto-phonons, which are not well separated in energy for these small values of $N$. This decomposition in families is first understood in the case of a quasi-particle over the Laughlin state $(\nu<1 / 3)$ : we find a precise correspondence between the exact spectrum and the conformal field theory by using the Jain composite-fermion theory, as it follows.

We begin by computing the overlap matrix between the exact low-lying states and the Jain states of the branch (for example, suppose that the quasi-particle corresponds to the filling [6,2] for eight electrons). These Jain states are particle-hole excitations of two effective Landau levels, and can be mapped one-to-one to the states of the twocomponent Abelian conformal theory. As a consequence, each low-lying exact state in the branch has a label of this conformal theory (up to $O(1 / N)$ finite-size errors). We then find that the edge excitations of the Laughlin quasiparticle match certain conformal states which are symmetric with respect to the two Abelian components, i.e. the two effective Landau levels. More precisely, these states are obtained by the projection $\widehat{U(1)} \times \widehat{U(1)} \rightarrow \widehat{U(1)}$ diagonal relating the two-component and the one-component Abelian theories. Therefore, the Laughlin quasi-particles are indeed described by the one-component Abelian theory, like the well-understood quasi-holes - this is expected in a relativistic theory. Moreover, the quasi-particle edge excitations amount to a specific sub-set of the larger Jain spectrum.

Having understood the pattern of the edge excitations of the Laughlin quasi-particles, we proceed to analyse other branches in the spectrum, whose edge excitations are less understood. For eight electrons, we find that the edge spectrum of the $\nu=2 / 5$ hierarchical Hall state and its first quasi-particle match the predictions of the $W_{1+\infty}$ minimal conformal theory. This theory realizes the weaker projection $\widehat{U(1)} \times \widehat{U(1)} \rightarrow \widehat{U(1)}$ diagonal $\times$ Virasoro $[18$, which again 
eliminates the antisymmetric excitations between the two Abelian components, but keeps all the symmetric ones [25]; there remains a non-trivial sector of neutral excitations, as explained in Section IIC. Therefore, the numerical study support the description of the hierarchical plateaus by the $W_{1+\infty}$ minimal conformal theory. The electrons form an irreducible, minimal incompressible fluid, which is in many respects analogous to the original Laughlin fluid [3].

The same pattern of families of edge excitations in the low-lying spectrum is also found for $N=6$ and 10 electrons. For $N=6$, the finite-size span for the angular momentum of edge excitations, $\Delta J<O(\sqrt{N})$, is too small to see any difference between the one-component Abelian (Laughlin states) and the $W_{1+\infty}$ minimal theories (hierarchical states); namely, the results are consistent with our picture but are not very significative. For $N=10$, we find that the $\nu=2 / 5$ Hall plateau clearly displays the edge spectrum predicted by the $W_{1+\infty}$ minimal theory, as in the $N=8$ case; on the other hand, the $\nu=3 / 7$ hierarchical state and its quasi-particles are less neat. Anyhow, these data cannot be consistently interpreted by the alternative multi-component Abelian theory.

The paper is organized as follows. In Section 2, we recall the main results of the Jain theory and the predictions of the two classes of CFTs, which are then used to analyse the data in Section $3(N=8)$ and Section $4(N=6,10)$. Finally, we end up with some Conclusions.

\section{THE NUMERICAL EXPERIMENT AND ITS THEORETICAL INTERPRETATIONS}

We consider a system of $N=6,8,10$ electrons in the first Landau level on a disk geometry; we use open boundary conditions, namely, we truncate the Hilbert space of single-particle angular momentum states at a value that is very large compared to the highest occupied level in the Laughlin state. The interaction among the electrons is given by the Haldane short-range potential, which selects the $\nu=1 / 3$ Laughlin incompressible ground state. Of course, we are interested in studying the $\nu=2 / 5$ incompressible ground state, and other hierarchical states, for which there is no analogous model interaction ${ }^{3}$.

An overview of the $N=8$ spectrum is shown in Fig.(1) as a function of the angular momentum $J$ : one clearly sees the branches of low-lying states. No confining potential is present, but this can be easily added afterwards: a quadratic potential increases the energy of each state by an amount proportional to $J$; thus, the bottom state of each branch can be made the ground state by suitably tuning the strength of the potential. If this ground state has an approximately constant density, its filling fraction is $\nu \sim N(N-1) / 2 J$, up to finite-size corrections of relative order $O(1 / N)$. Let us finally quote the previous analysis on the disk geometry, which were useful in setting up our work [9] [23] [24].

\section{A. The Jain Hierarchy}

According to the composite-fermion theory, there is a correspondence between $m$ filled effective Landau levels $\left(\nu^{*}=m\right)$ and the hierarchical Hall states [幽:

$$
\nu^{*}=m \quad \longleftrightarrow \quad \nu=\frac{m}{p m \pm 1}, \quad m=1,2, \ldots, \quad p=2,4, \ldots
$$

This correspondence is made explicit by the ansatz wave-functions

$$
\Psi_{\nu}\left(z_{1}, \ldots, z_{N}\right)=\mathcal{P}\left(\prod_{i<j}^{N}\left(z_{i}-z_{j}\right)^{2} \Psi_{\nu^{*}}\left(z_{1}, \ldots, z_{N}\right)\right)
$$

where $\Psi_{\nu^{*}}$ is the Slater determinant of the filled Landau levels and $\mathcal{P}$ is the projector into the first Landau level. The composite-fermion correspondence also describes the excited states by inserting in (2) the Slater determinants for the $\nu^{*}=m$ electron transitions.

On the disk geometry, the Landau levels can be filled selectively by making electron droplets with different edge shapes: these "bottom" states are denoted by $\left[n_{1}, n_{2}, \ldots, n_{k}\right]$ and correspond to the Slater determinants of $n_{i}$ electrons filling the lowest angular momentum states of the $i$-th Landau level [23]. Each bottom state has an independent branch of low-lying states corresponding to the particle-hole excitations of the effective levels. The Jain correspondence (2)

\footnotetext{
${ }^{3}$ For six electrons, we have also computed the spectrum for the Coulomb interaction and found the same qualitative features.
} 
applied to these bottom states yields $\nu<1$ states which are characterized by the following angular momenta and approximate energies [23]:

$$
\begin{aligned}
& J_{\left[n_{1}, \ldots, n_{k}\right]}=N(N-1)+\frac{n_{1}\left(n_{1}-1\right)}{2}+\frac{n_{2}\left(n_{2}-3\right)}{2}+\frac{n_{3}\left(n_{3}-5\right)}{2}+\cdots, \\
& E_{\left[n_{1}, \ldots, n_{k}\right]}^{(0)}=E_{D}\left(n_{2}+2 n_{3}+3 n_{4}+\cdots\right) .
\end{aligned}
$$

In the last equation, $E_{D}$ is the effective Landau level gap, which has been recently interpreted as the energy for creating a "defect" in the electron fluid [ 8]. In this work, the Jain ansatz was also modified to be completely written in the first Landau level; nevertheless, in this paper we shall use the original proposal (2) and perform the projection $\mathcal{P}$ numerically.

The Jain bottom states (3) for eight electrons are summarized in Table (I): the [8] state is identified as the Laughlin $\nu=1 / 3$ incompressible state. The rest of states could be candidates for the $\nu=2 / 5$ ground state, because they have $J \sim 70$ up to $O(N)$ finite-size corrections; actually, in the thermodynamic limit $N \rightarrow \infty$, any state $[N / 2+k, N / 2-k]$, with $k$ fixed, would work. The Jain bottom states match rather well the numerical spectrum in Fig.(11): for almost all predicted $J$ values in Table (I) , there is a bottom state of a branch of low-lying states; its energy is rather well approximated by the Jain formula (3). On the other hand, a disagreement is seen for the smaller $J$ states [4,4] and $[5,2,1]$, which have $E^{(0)}=4$ but are almost degenerate with the $E^{(0)}=3$ states; moreover, these two states seem to describe the same branch of levels - we shall discuss these points later on.

\section{B. Abelian Conformal Field Theories of Edge Excitations}

It is rather well established that the low-energy excitations of a droplet of quantum incompressible fluid reside on the boundary and can be described by a $(1+1)$-dimensional conformal field theory [9]. This can be simply understood in the case of one filled Landau level (see Fig.(2)), which can be considered as the Fermi sea (in configuration space) of a one-dimensional system with one chirality only [15]. Its low-lying excitations are particle-hole transitions near the Fermi surface, which is actually the physical edge of the (circular) electron droplet. By a well-known procedure, one can take the thermodynamic limit $N \rightarrow \infty$ and approximate the Fermi sea by a Dirac vacuum; moreover, the energy of the low-lying excitations can be linearized around the Fermi level, $\epsilon \sim v k$, where $k=2 \pi n / R$ is the momentum, $v$ the Fermi velocity and $R=O(\ell \sqrt{N / \nu})$ the size of the disk. These are the edge excitations of the $\nu=1$ quantum Hall state; in a finite system, their wave number is limited by $|n| \ll R$, i.e. their angular momentum must be $\Delta J \ll O(\sqrt{N})$ [16]. Besides the edge excitations, there are quasi-hole excitations which amount to moving one electron from deep inside the Fermi sea to the edge $(\Delta J=O(N))$, and, conversely, for the quasi-particles.

In the thermodynamic limit, the relativistic one-dimensional effective fermion is identified as the charged, chiral Weyl fermion; the conformal symmetry of this theory is described by the Virasoro algebra with central charge $c=1$ [14. This effective conformal field theory of the $\nu=1$ plateau can be generalized to the Laughlin states by the wellknown bosonization procedure: one rewrites the Weyl fermion in terms of a bosonic field, changes its compactification radius and obtains a general $c=1$ Abelian conformal theory, whose one-dimensional current satisfies the Abelian current algebra $\widehat{U(1)}[16]$; an equivalent name is the chiral Luttinger liquid [9]. This description of the edge excitations of the Laughlin states is well established both theoretically and experimentally [10] 111] [12].

An important property for the following discussion is that the Hilbert spaces of the edge excitations of the integer and Laughlin Hall states, $\nu=1,1 / 3,1 / 5, \ldots$, are all isomorphic [9]; thus, these excitations can be still visualized as the particle-hole transitions in Fig.(2). It is rather simple to count them for small values of $\Delta J=n$, and obtain the multiplicities reported in the first line of Table (II] [9]; let us stress that the CFT description of edge excitations is valid in the linear range $\Delta J<O(\sqrt{N})$ 16.

A natural generalization of the above picture is to consider the case of two filled Landau levels: the corresponding edge excitations are described by the two-component Abelian CFT, which is simply the tensor product of two onecomponent theories. Each Landau level has its own Fermi surface and its particle-hole excitations; their total number is obtained by adding the excitations of the two levels which have a given $\Delta J=\Delta J_{1}+\Delta J_{2}$ (see the second row of Table (III).

According to the Jain composite-fermion correspondence (1), it is rather natural to use this two-component Abelian theory for describing the edge excitations of the hierarchical states (2) with $\nu=2 /(2 p+1)=2 / 5,2 / 9, \ldots$ As in the

\footnotetext{
${ }^{4}$ The interaction among defects is clearly neglected in $E^{(0)}$.
} 
one-component case, these $\nu<2$ values can be described in the conformal theory by bosonizing the two Weyl fermions and by suitably changing their compactification radii [9]. A characteristic feature of the resulting conformal theories is to realize an extension of the Abelian current algebra symmetry $\widehat{U(1)} \otimes \widehat{U(1)} \rightarrow \widehat{U(1)} \otimes \widehat{S U(2)}$, 17 [ [13; ; this will be useful in the following discussion. As in the one-component case, the Hilbert spaces of the edge excitations above the $\nu=2$ Hall plateau and all the $m=2$ hierarchical states (2) are isomorphic.

\section{Minimal Models of Edge Excitations}

Another theory of the hierarchical edge excitations has been independently proposed in the Ref. [18]: it corresponds to the one-component Abelian CFT for the simplest Laughlin fluids, but differs from the multi-component theory. It is based on the physical picture of the incompressible fluid [3], which possesses the dynamical symmetry under the area-preserving diffeomorphisms of the spatial coordinates 20] [19]. This symmetry has been promoted to a building principle for the CFTs describing its edge excitations [21]: actually, it implies that the conformal fields should carry a representation of the $W_{1+\infty}$ algebra, which is a generalization of the Abelian current algebra [26].

Among the conformal theories with $W_{1+\infty}$ algebra [21], a particular class of models has been found, the $W_{1+\infty}$ minimal models [18], whose filling fractions are in one-to-one correspondence with the hierarchical values (11). This is already a strong indication that these models are experimentally relevant. The minimal models are characterized by being a reduction of the previous multi-component Abelian CFTs, in the sense that some excitations are projected out, as explained hereafter. This projection implies [18]: (i) a reduced number of edge excitations above the ground state, as given in the third line of Table (II); (ii) only one independent Abelian charge for the quasi-particles, which is hence identified with the electric charge; (iii) the existence of neutral quasi-particle excitations characterized by a non-Abelian quantum number of the $S U(m)$ Lie algebra, where $m$ is the number of would-be components.

This projection has been recently made explicit by a Hamiltonian formulation of the minimal incompressible models [25], which will be briefly summarized in the first non-trivial case of two components $(m=c=2)$. Let us start from a closer look into the two-component Abelian theory with symmetry $\widehat{U(1)} \otimes \widehat{S U(2)})_{1}$; for $\nu=2$, this is described by two Weyl fermions $\Psi_{i}(\theta), \bar{\Psi}_{i}(\theta)$, where $\theta$ is the angular variable on the disk and $i=1,2$ denote the upper and lower levels, respectively. Their excitations can be labelled by the total Abelian charge $J_{0}$ and the $S U(2)$ isospin charge $J_{0}^{3}$, which are defined as follows:

$$
\begin{aligned}
J_{0} & =\int_{0}^{2 \pi} \frac{d \theta}{2 \pi}\left(\bar{\Psi}_{1} \Psi_{1}+\bar{\Psi}_{2} \Psi_{2}\right) \\
J_{0}^{3} & =\int_{0}^{2 \pi} \frac{d \theta}{2 \pi} \frac{1}{2}\left(\bar{\Psi}_{1} \Psi_{1}-\bar{\Psi}_{2} \Psi_{2}\right), \\
J_{0}^{+} & =\int_{0}^{2 \pi} \frac{d \theta}{2 \pi} \bar{\Psi}_{1} \Psi_{2}, \quad J_{0}^{-}=\int_{0}^{2 \pi} \frac{d \theta}{2 \pi} \bar{\Psi}_{2} \Psi_{1} .
\end{aligned}
$$

One can check that $\left\{J_{0}^{+}, J_{0}^{-}, J_{0}^{3}\right\}$ satisfy the $S U(2)$ algebra by using the fermionic canonical commutation relations. The Abelian and iso-spin charges measure the edge excitations in the two layers symmetrically and anti-symmetrically, respectively. Note, however, that the quasi-particles in these Abelian theories carry the iso-spin quantum number in such a way that it is linearly additive as another electric charge; namely, there are no non-Abelian effects [9]. These $S U(2)$ generators can be defined for all the hierarchical ground states $\nu=2 / 5,2 / 9, \ldots$, but are not realized in terms of fermions; we can nevertheless continue to use the more intuitive fermionic language, owing to the aforementioned isomorphism between Hilbert spaces.

After these preliminaries, we are ready to define the $W_{1+\infty}$ minimal theory (for $c=2$ ): this is obtained from the two-component Abelian theory by imposing the constraint [25],

$$
\left.J_{0}^{-} \mid \text {minimal states }\right\rangle=0 \text {. }
$$

It can be shown that the zero modes $\left\{J_{0}^{ \pm}, J_{0}^{3}\right\}$ commute with the Virasoro generators; thus, the constraint (5) does not spoil the conformal invariance and defines a new conformal theory with the same central charge.

The effect of the constraint is the following: the operator $J_{0}^{-}$moves electrons down and holes up between the two layers (with a minus sign in the latter case), while keeping their (normal-ordered) angular momentum constant; it

${ }^{5}$ The full set of Fourier modes $\left\{J_{n}^{a}\right\}$ generate the current algebra $\left.\widehat{S U(2)}\right)_{1}$ which contains this $S U(2)$ as a subalgebra 14$]$. 
relates the edge excitations in the two layers and actually vanishes on their symmetric linear combinations. Therefore, the condition (5) projects out the edge excitations which are antisymmetric with respect to the two levels. The ground state is unique and symmetric, then it satisfies the constraint: namely, the two CFTs share the same ground state.

Let us see some examples of allowed edge excitations in the minimal theory. We first need to clarify the angularmomentum labels in CFT. The Weyl fermions define a second-quantized relativistic theory, which describes excitations above the ground state; therefore, the latter must be specified in order to relate this description to the numerical data. For example, one can suppose that the $N=8$ bottom state $[5,3]$ with $J=66$ is the $\nu=2 / 5$ incompressible ground state (see Fig.(11)). This fixes the Fermi surface for both layers and sets the reference value for the angular momentum of excitations: $\Delta J_{1}=J_{1}-4$ and $\Delta J_{2}=J_{2}-1$. The usual moding of conformal fields thus corresponds to the normal-ordered angular momentum, which is equal to zero for the charges in (14). The ground state [5,3] and its edge excitations are identified by vanishing eigenvalues of both $J_{0}$ and $J_{0}^{3}$; the other Jain branches in the spectrum, corresponding to $[6,2]$, etc., in Table(II), are described by the CFT as excitations with $J_{0}^{3}=-1$, etc, respectively.

Let us now introduce the fermionic Fock space of the two Weyl fermions (14), which also describe the particle-hole excitations of the two effective Landau levels, "up" and "down", in the Jain construction: the fermionic secondquantized operators are, respectively, $u_{k}, u_{k}^{\dagger}$ and $d_{k}, d_{k}^{\dagger}(k \in \mathbf{Z})$; they act on the ground state $|\Omega\rangle$. There are two Abelian edge excitations at the first excited level $\Delta J=1$, which can be written:

$$
|1 ; \pm\rangle=\frac{1}{\sqrt{2}}\left(d_{1}^{\dagger} d_{0}|\Omega\rangle \pm u_{1}^{\dagger} u_{0}|\Omega\rangle\right)
$$

The constraint (5) can be written explicitly: $J_{0}^{-}|1 ; \pm\rangle=\sum_{k=-\infty}^{\infty} d_{k}^{\dagger} u_{k}|1 ; \pm\rangle=0$. We find that the symmetric combination $|1 ;+\rangle$ satisfies this constraint and the antisymmetric does not: therefore, the $W_{1+\infty}$ minimal conformal theory only contains the symmetric excitations, as we anticipated. At the next level $\Delta J=2$, there are five Abelian edge states:

$$
\begin{aligned}
|2 ; a\rangle & =d_{1}^{\dagger} d_{0} u_{1}^{\dagger} u_{0}|\Omega\rangle, \\
|2 ; b \pm\rangle & =\frac{1}{\sqrt{2}}\left(d_{2}^{\dagger} d_{0}|\Omega\rangle \pm u_{2}^{\dagger} u_{0}|\Omega\rangle\right), \\
|2 ; c \pm\rangle & =\frac{1}{\sqrt{2}}\left(d_{1}^{\dagger} d_{-1}|\Omega\rangle \pm u_{1}^{\dagger} u_{-1}|\Omega\rangle\right) .
\end{aligned}
$$

The antisymmetric combinations $|2 ; b-\rangle$ and $|2 ; c-\rangle$ do not satisfy the constraint (5) and are not present in the minimal CFT. Note that the counting of these states is in agreement with Table (II).

In the semiclassical picture developed in Ref. [20], the incompressible Hall fluid is identified with a Fermi sea and its area-preserving deformations are the particle-hole excitations (Fig.(22)). The two-level structure introduces one additional degree of freedom: the antisymmetric excitations are tangential to the Fermi surface and do not correspond to deformations of the incompressible fluid; therefore, they need not to be included in a minimal theory. This is the physical meaning of the condition (5).

A precise derivation of this constraint can be obtained by analysing the irreducible representations of the $W_{1+\infty}$ algebra. A general property of CFTs is that they can be constructed by assembling the representations of their infinite-dimensional symmetry algebra. The $W_{1+\infty}$ minimal theories were first obtained in such a way [18], by using the special, degenerate $W_{1+\infty}$ representations, which are equivalent to those of the algebra $\widehat{U(1)} \times \mathcal{W}_{m}$, in particular $\widehat{U(1)} \times$ Virasoro for $m=2$ [26]. The constraint (5) precisely realizes the projection of conformal theories $\widehat{U(1)} \times \widehat{S U(2)_{1}} \rightarrow \widehat{U(1)} \times$ Virasoro [25], which is a simplified case of the general mechanism of Hamiltonian reduction 27.

Note that the projection only keeps one state for each $S U(2)$ multiplet in the Abelian spectrum, i.e. the highestweight state; these states form a non-trivial set of neutral edge excitations, which are characterized by their Virasoro weight. The edge excitations of the other, $\widehat{U(1)}$ diagonal part are generated by the modes of the symmetric current in (画),

$$
J_{-k}=\sum_{j=-\infty}^{\infty} d_{k-j}^{\dagger} d_{j}+u_{k-j}^{\dagger} u_{j}, \quad k=1,2, \ldots
$$

These excitations are symmetric with respect to the two Landau levels, but are not the most general ones: for example, the state $|2 ; a\rangle$ in (7) cannot be obtained by applying (8) on the ground state. The symmetric edge excitations obtained by (8) realize the reduction of the two-component Abelian theory to the one-component theory $\widehat{U(1)} \times \widehat{U(1)} \rightarrow \widehat{U(1)}$ diagonal, which also entails a change of central charge $c=2 \rightarrow 1$. 
Finally, we remark that the constraint (5) can be enforced dynamically by modifying the Hamiltonian of the two-component Abelian theory:

$$
H \rightarrow H+\gamma J_{0}^{+} J_{0}^{-}
$$

The added term is diagonal in the two-component Abelian Hilbert space, and is relevant in the renormalization-group sense, because $\gamma$ has dimension of a mass. It increases the energy of the states which do not satisfy (5) and in the limit $\gamma \rightarrow \infty$, it performs the projection leading to the $W_{1+\infty}$ minimal model. In general, we may also consider the non-conformal theory with $\gamma \neq 0, \infty$, which interpolates between the two-component and the minimal, i.e. irreducible, incompressible fluids . A more complete discussion of these matters can be found in Ref. [25].

\section{ANALYSIS OF THE $N=8$ DATA}

We now proceed to analyse each branch of levels in the $N=8$ spectrum and interpret it according to the theories of the previous Section. The exact eigenstates are denoted by $\| J-n\rangle\rangle$, where $J$ is the angular momentum and $n=0,1,2, \ldots$ the ordering by increasing values of the energy. The complete set of our numerical data is accessible on-line7.

\section{Branch [8]: the Laughlin incompressible Hall fluid at $\nu=1 / 3$}

The density profile $\rho(\mathbf{x})$ of the Laughlin ground state $\| 84-0\rangle\rangle$ is drawn in Fig.(3): we see that this droplet of incompressible fluid is fairly flat in the interior; moreover, its value at the origin $2 \pi \rho(\mathbf{0})$ is close to the expected value of $1 / 3$ in the thermodynamic limit. The edge excitations are recognized as very small deformations of the shape of the ground state density; their energies above the ground state vanish for the Haldane interaction, as shown in the inset of Fig.(3). Having identified the edge states, we can count their number: we find the multiplicities $(1,1,2,3, \ldots)$ for $\Delta J=(0,1,2,3, \ldots)$, in agreement with the predictions of the one-component Abelian CFT in Table(这). The matching of the exact edge excitations with the the corresponding Jain (Laughlin) wave functions is rather obvious in this case; the total overlap with each exact numerical state is one by construction.

\section{Branches $[7,1],[6,2]$ : the quasi-particles over the Laughlin state}

Fig.(11) shows the density profiles of all the bottom states found in the $N=8$ spectrum: these are all more oscillating than the Laughlin ground state. The states $\| 63-0\rangle\rangle$ and $\| 70-0\rangle\rangle$ are clearly quasi-particle excitations with the characteristic bump of size $O(\ell)$ : therefore, they cannot be interpreted as other incompressible ground states with $\nu<1 / 3$. Let us analyse the $[6,2]$ branch $J=70$ in more detail: the density profiles and energies of its low-lying states are drawn in Fig.(5). One distinguishes two families of states with opposite oscillations, which have multiplicities $(1,1,2)$ and $(0,1,3)$, respectively. The first group can be interpreted as a quasi-particle on top of the Laughlin $\nu=1 / 3$ ground state: actually, the multiplicities of its edge excitations agree with the predictions of the one-component Abelian conformal theory (Table (II)). The second group can be interpreted as another bulk excitation, which might acquire a larger gap in the thermodynamic limit, where the CFT description of the low-lying states should become exact. Although this limit cannot be inferred from the simulation, the inset of magnified energy levels in Fig.(5) clearly shows that these states have a higher energy than those of the previous group.

Note that the sum of the degeneracies of the two groups considered above match the predictions of the twocomponent Abelian CFT in Table (III), i.e. $(1,2,5)$. By ignoring the information arising from the density plots, one might be tempted to interpret all these low-lying states as edge excitations; then, the corresponding bottom state could not be a Laughlin quasi-particle, because the edge multiplicities would not match; it would rather be interpreted as the $\nu=2 / 5$ incompressible fluid [9]. However, the $J=76$ branch, i.e. [7,1], being qualitatively similar, should be interpreted in the same way, while it is necessarily a Laughlin quasi-particle, thus arriving to a contradiction. We conclude that the new criterion of density plots is essential for distinguishing Laughlin quasi-particles from new hierarchical plateaus. We can actually observe the formation of a hierarchical Hall fluid [28]: as soon as sizable number

\footnotetext{
6 These are the repulsive and attractive fixed points of the renormalization group trajectory, respectively.

7 See: http://andrea.fi.infn.it/cappelli/disk.htm.

${ }^{8}$ Hereafter, we set the magnetic length $\ell=1$.
} 
$O(N / 2)$ of Laughlin quasi-particles are present, they condense into a new incompressible fluid, whose density shape is again smooth (see next paragraph).

After having established that $J=70$ is a Laughlin quasi-particle, we should understand the origin of the extra family $(0,1,3)$ of low-lying states. This is explained by the Jain theory, which we now analyse in detail; we are going to illustrate the following chain of relations:

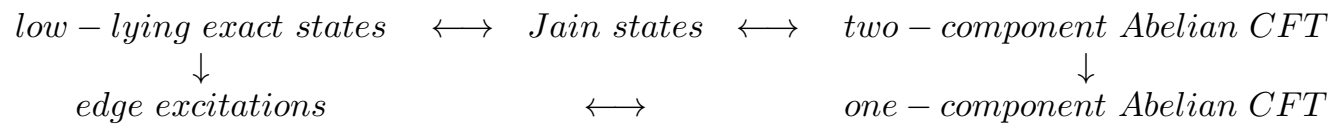

The Jain wave functions corresponding to the [6,2] branch are obtained by plugging in (2) the Slater determinants for the particle-hole excitations of the first and second levels filled with 6 and 2 electrons, respectively. These states are, by construction, in one-to-one correspondence with those of the two-component Abelian conformal theory; thus, we use the same notation for them, in particular the basis of Section IIC, equations (6) and (7). Note, however, that the Jain states are not orthogonal; therefore, we diagonalize them by the Gram-Schmidt method甲 (by also computing the overlaps among themselves).

For each angular momentum value $\Delta J=0,1,2$, we have computed the matrix of overlaps between these Jain states and the low-lying spectrum (see Table (III)). The total square overlap with each state is very large (about 0.96), and the matrix determinant is large enough to match these states one-to-one. We conclude that the Jain theory describes very well all the low-lying states. Nonetheless, this does not imply that they all should be edge excitations, as shown by the previous arguments of the density plot and of the consistency with the Laughlin theory. Let us recall that, in principle, the Jain theory and the conformal field theory give rather different descriptions of the spectrum: the former applies directly to all excitations for finite $N$, while the latter only accounts for the edge excitations in the large $N$ limit, which requires some imagination.

The analysis of the overlap matrices in Table ([II]) let us identify which Jain states correspond to the edge excitations of the Laughlin quasi-particle in Fig.(6). The edge state $\| 71-0\rangle\rangle$ clearly matches the symmetric state $|1 ;+\rangle$ in equation (6). The five states at $\Delta J=2$ can be divided in two groups: $(\| 72-0\rangle\rangle, \| 72-1\rangle\rangle, \| 72-3\rangle\rangle)$ have larger overlaps with the symmetric states in (7), and $(\| 72-2\rangle\rangle, \| 72-4\rangle\rangle)$ with the antisymmetric states. The precision 10 is lower than for $\Delta J=1$, but it is nonetheless remarkable that the signal is not completely washed out by the finite-size effects.

This pattern of overlaps can be understood as a relation among conformal field theories by applying the analysis of Section IIC: the edge excitations of the Laughlin quasi-particle, $\| 71-0\rangle\rangle, \| 72-0\rangle\rangle$ and $\| 72-1\rangle\rangle$, have large overlaps with the symmetric states of the two-component Abelian theory which are generated by the $\widehat{U(1)}$ diagonal current (8), namely $|1 ;+\rangle,|2 ; b+\rangle$ and $|2 ; c+\rangle$. Therefore, the Laughlin quasi-particle is indeed described by the onecomponent Abelian conformal theory; the new result is that this is obtained in the Jain spectrum by the projection $\widehat{U(1)} \times \widehat{U(1)} \rightarrow \widehat{U(1)}$ diagonal described in the Section IIC.

Similar families of symmetric Laughlin edge excitations with multiplicities $(1,1,2)$ are actually found in any branch and for any number of electrons: this is perhaps the most important result of this paper. These "standard" families are enlarged by further edge excitations in the case of hierarchical Hall states, as discussed hereafter.

\section{Branches $[\mathbf{5 , 3}],[\mathbf{5 , 2 , 1 ]}:$ the $\nu=2 / 5$ hierarchical Hall state and its quasi-particle}

Fig.(6) shows the family of low-lying states starting at $J=66$, which is identified with the [5,3] branch. The bottom state $\| 66-0\rangle\rangle$ has a rather flat profile and can be considered as a new incompressible ground state; the value of $2 \pi \rho(\mathbf{0})$ is close to its thermodynamic limit of $2 / 5$ (at variance with the quasi-particle state $\| 76-0\rangle\rangle$ ). Moreover, a family of edge excitations is clearly associated to $\| 66-0\rangle\rangle$, which have multiplicities $(1,1,3)$, in agreement with the predictions of the $W_{1+\infty}$ minimal CFT (third row of Table (III)).

Next, we check whether these edge excitations match the states of the minimal theory, by analysing the overlaps with the Jain states (see Table (IV)). We find that the ground state $\| 66-0\rangle\rangle$ is well-approximated by the Jain bottom state $|[5,3]\rangle$. For $\Delta J=1$, the unique edge state $\| 67-0\rangle\rangle$ is again identified with the symmetric Jain state $|1 ;+\rangle$. At $\Delta J=2$, the exact edge states $\| 68-i\rangle\rangle$, with $i=1,2,3$ have large overlaps with the symmetric Jain states $|2 ; a\rangle,|2 ; b+\rangle$ and $|2 ; c+\rangle$, and $O(1 / N)$ projection on the antisymmetric Jain states. Therefore, the edge excitations match all the possible symmetric Jain states; according to Section IIC, these are singled out by the projection $\widehat{U(1)} \times \widehat{U(1)} \rightarrow \widehat{U(1)} \times$ Virasoro, leading to the $W_{1+\infty}$ minimal theory.

\footnotetext{
9 This introduces some ambiguities for the ordering of states, but they do not affect the qualitative properties to be discussed hereafter.

10 Table (III) reports approximate numbers for an easy reading; precise data can be found on-line.
} 
Putting all these informations together, we conclude that this branch can be definitely interpreted as the $\nu=2 / 5$ incompressible fluid for $N=8$ electrons, and that its edge excitations are described by the $W_{1+\infty}$ minimal conformal theory [18] within the finite-size accuracy.

Let us add one remark. The third state $\| 68-0\rangle\rangle$ at $\Delta J=2$ which makes the difference between the ubiquitous Laughlin edge spectrum and the hierarchical one, has an alternative description in the Jain theory as the bottom state of the $[6,1,1]$ branch. Here we first encounter the phenomenon of superposition of Jain branches, which is a sort of degeneracy in the description of the spectrum; it is an interesting dynamical problem in the Jain theory, which has not been addressed so far. It can be rephrased as an "interaction among defects" in the regime of compact electron droplets [4]; it might also be associated to the mechanism of the "condensation of quasi-particles" of the original hierarchical scheme [28] (or maybe not). As a matter of fact, this dynamical phenomena does not concern the CFT interpretation, which is an effective description of the edge excitations above a given ground state; there is just a practical consequence that the one-to-one identification of states in (10) might be lost in case of mixing of two competing Jain branches.

The overlaps of the $[6,1,1]$ Jain branch with the $J=68,69$ low-lying states are not very good, although the bottom state itself has a good overlap. This branch is rather atypical, and cannot be considered as a candidate incompressible state because it is almost gapless with the $[5,3]$ branch.

We now proceed to analyse the branch $J=63$, which is plotted in Fig.(7). Its natural interpretation is of a quasiparticle over the $\nu=2 / 5$ ground state, due to the oscillating density profile. The $W_{1+\infty}$ minimal theory predicts the same pattern of edge excitations as for the $\nu=2 / 5$ ground state, i.e. $(1,1,3)$ [18]; this is actually observed in Fig.(17), and add further support to whole picture put forward in this paper. A slight peculiarity is that the third state at $\Delta J=2$ is higher in energy than other non-edge states - but the shape is more important in our analysis.

Next, we proceed to the identification of these states by the overlap analysis (see Table $(\overline{\mathrm{VII}})$ ). The Jain theory presents a degenerate description for this branch, namely $[4,4]$ and $[5,2,1]$ (See Table (ID)). The overlaps show that the latter wins, i.e. the states of the $[4,4]$ branch have less than $O(1 / N)$ projection on the low-lying states; apparently, the electron tends to "pile up" in the effective Landau levels. The empirical rule taken from this and other cases of two competing Jain branches is that one of them describes well the data, while the other definitely does not.

The Jain states of the $[5,2,1]$ branch should be related to a three-component Abelian conformal theory; this is the first step in extending the chain of relations (10) to this branch. For $\Delta J=1$, there are three Abelian edge states, which can be written, in analogy with Eq.(5):

$$
\begin{aligned}
& |1 ; a\rangle=\frac{1}{\sqrt{3}}\left(u_{1}^{\dagger} u_{0}|\Omega\rangle+c_{1}^{\dagger} c_{0}|\Omega\rangle+d_{1}^{\dagger} d_{0}|\Omega\rangle\right), \\
& |1 ; b\rangle=\frac{1}{\sqrt{2}}\left(u_{1}^{\dagger} u_{0}|\Omega\rangle-c_{1}^{\dagger} c_{0}|\Omega\rangle\right), \\
& |1 ; c\rangle=\frac{1}{\sqrt{6}}\left(u_{1}^{\dagger} u_{0}|\Omega\rangle+c_{1}^{\dagger} c_{0}|\Omega\rangle-2 d_{1}^{\dagger} d_{0}|\Omega\rangle\right) .
\end{aligned}
$$

The ground state is $|\Omega\rangle=|[5,2,1]\rangle$ and the $\left\{u_{k}, c_{k}, d_{k}\right\}$ are fermionic Fock operators for the upper, central and lower (effective) Landau levels.

In this case, we expect that the edge excitations are described by a reduction from the three-component Abelian theory to the $c=2 W_{1+\infty}$ minimal theory (analogous to the $c=2 \rightarrow 1$ projection (10) for the Laughlin quasiparticles). It can be shown [25] that this reduction implies that the unique edge excitation at $\Delta J=1$ is the completely symmetric state $|1 ; a\rangle$. This is in agreement with the overlaps reported in Table (V).

Next, the overlap analysis cannot be carried over to the $\Delta J=2$ edge excitations, owing to the finite-size limitations: the Jain state $[5,2,1]$ possesses only one electron in the highest effective Landau level, and is very far from the thermodynamic limit of three Fermi surfaces which is implicit in the CFT description; in particular, one of the $\Delta J=2$ particle-hole excitations in the higher level is missing. Therefore, we cannot analyse the projection of states in this branch; nevertheless, the shape and number of edge excitations are in agreement with the predictions of the $W_{1+\infty}$ minimal theory for a quasi-particle over the $\nu=2 / 5$ state.

\section{ANALYSIS OF THE $N=6$ AND 10 DATA}

\section{A. The $N=6$ Data}

Fig. (8) shows the exact spectrum as a function of the angular momentum and Table (VI) reports the bottom states of the Jain branches with $J \geq 33$. The observed structure in branches is similar to that of the $N=8$ case: 
the Laughlin branch starts at $J=45$, and its ground state is identified with the $[6,0]$ Jain state. In decreasing order of $J$, we identify as $[5,1]$ the state appearing at $J=39$ and as $[4,2]$ the one at $J=35$. For the state appearing at $J=33$ there are two possible Jain states, $[3,3]$ and $[4,1,1]$, which have the same energy $E^{(0)}$. Figure $(9)$ shows the density profiles of all the bottom states: these give the first hints for identifying the incompressible ground states. The profiles should be compared with the data of average density $\rho(0)$ and angular momentum for idealized flat droplets, which are reported in Table (VII). One finds that the bottom states with $J=39$ and $J=33$ are candidates for the incompressible Hall states with $\nu=2 / 5$ and $3 / 7$, respectively. Let us analyse them in turn.

The profiles of the low-lying states of the $J=39$ branch are shown in Fig.(10): the edge states are $\| 40-0\rangle\rangle$, $\| 41-0\rangle\rangle$ and $\| 41-1\rangle\rangle$, i.e. the observed multiplicities are $(1,1,2)$. The analysis of the overlaps with the Jain wave functions is similar to the previous cases: the results are that the bottom state $\| 39-0\rangle\rangle$ is identified with $|[5,1]\rangle$, as expected; for $\Delta J=1$, the edge state $\| 40-0\rangle\rangle$ is the symmetric combination of Abelian edge states $|1 ;+\rangle$ (see Eq.(6)); instead, the non-edge eigenstate is || $40-1\rangle\rangle \sim|1 ;-\rangle$. The overlap analysis cannot be extended to $\Delta J=2$, because the $[5,1]$ ground state has only one electron in the second effective Landau level, and its particle-hole excitations cannot match the two-component Abelian CFT. Within the $\Delta J=1$ analysis, we cannot distinguish the minimal $c=2$ edge (multiplicities $(1,1,3, \ldots))$ from the $c=1$ Abelian edge $(1,1,2, \ldots)$ associated with the Laughlin quasi-particle excitations. In conclusion, this branch can be interpreted either as the $\nu=2 / 5$ incompressible Hall state described by the $W_{1+\infty}$ minimal theory (but one edge excitation is missing), or as a Laughlin quasi-particle (but its profile is exceptionally flat).

The density profiles of the $J=33$ branch are presented in Fig. (11): the edge excitations are $\| 34-0\rangle\rangle, \| 35-1\rangle\rangle$ and $\| 35-2\rangle\rangle$, i.e. again Laughlin-like multiplicities. Their interpretation in conformal field theory begins by identifying the ground state $\| 33-0\rangle\rangle$ with one of the two possible Jain bottom states: the overlaps in Table (VIII) shows that the exact state is well described by the three-level state $[4,1,1]$, rather than the "simpler" $[3,3]$ one; this is the "piling-up" of the electrons, which we have already encountered. Actually, the computation of the energies of the two Jain bottom states shows that the $[3,3]$ branch is separated from the low-lying $[4,1,1]$ branch by a gap of order $E_{D}$; in this case, the interaction among defects is of the same order as the energy $E^{(0)}$ for non-interacting defects 田.

Next, we analyse the low-lying states of this branch: the Jain excitations of the $[4,1,1]$ bottom state should be compared with the three-component Abelian CFT, as we have already done for the branch $[5,2,1]$ of $N=8$ electrons. Again, we must limit ourselves to the excitations with $\Delta J=1$, due to the finite sizes of the would-be Fermi seas. The results for the overlaps in Table (VIII) are very similar to those in Table (V) for the quasi-particle over the $\nu=2 / 5$ state: the unique edge state $\| 34-0\rangle\rangle$ is clearly identified as the symmetric excitation $|1 ; a\rangle$.

The density shape of the $J=33$ bottom state suggests its interpretation as the $\nu=3 / 7$ hierarchical Hall state for $N=6$ electrons. Then, we expect that its edge excitations are described by the $c=3 W_{1+\infty}$ minimal conformal theory, whose multiplicities are again $(1,1,3)$ (see the last row of Table (III) [18]; actually, one can show that the excitations of the $c=3$ and $c=2$ minimal theories only differ for $\Delta J \geq 3$, and that both agree with those of the simpler Laughlin theory for $\Delta J=1$.

In conclusion, the $N=6$ exact edge states are consistently described by the $W_{1+\infty}$ minimal conformal theory [18], within the finite-size limitations: we recall that the span for edge excitations in CFT is $\Delta J<O(\sqrt{N}) \sim 2$ [16]. Another consistent interpretation for all the branches is given by the Laughlin one-component theory; possibly, the condensation of quasi-particles leading to the hierarchical Hall fluids cannot take place in such a small system. Nevertheless, it is important that the $N=6$ and the $N=8$ data can be consistently interpreted.

\section{B. The $N=10$ Data}

The spectrum of energies as a function of the angular momentum for $N=10$ is presented in Fig. (12) and the branches of Jain states are given in Table (IX); the Laughlin ground state $[10]$ and the $[9,1]$ branch are not presented in Fig. (12), which focusses on the $\nu \leq 2 / 5$ region. The comparison between the exact branches and the Jain predictions shows the dynamical phenomenon already seen before: for any pair of Jain branches which have degenerate energy $E^{(0)}$ (Table $(\sqrt{\mathrm{IX}})$ ), only one is realized in the spectrum, and correctly describes the low-lying states (as shown by the overlap analysis). Actually, $J=111$ matches $[7,3]$, but $[8,1,1]$ is not observed; similarly $J=108$ matches $[7,2,1]$, $J=103$ is $[6,3,1]$ and $J=101$ is $[6,2,2]$, while $[6,4],[5,5]$ and $[5,4,1]$ are not observed; these are new examples of the "piling up" effect.

The analysis of the density profiles of all the bottom states in Fig.(13) shows that the branches $J=125$ and $J=117$ are quasi-particles, with the same qualitative features of the analogous $N=8$ cases. The bottom states of the $J=111$ and 103 branches are not growing in the bulk: although rather oscillating, they are the possible candidates for the $\nu=2 / 5$ and $\nu=3 / 7$ incompressible Hall states with $N=10$ electrons, respectively (see the data in Table (VII)).

The by-now standard analysis of the edge excitations is first performed on the $J=111$ branch; the plots are shown 
in Fig.(14) and the overlaps with the Jain states are reported in Table ( $\mathbb{X}$ ). The usual Laughlin edge excitations $(\| 112-0\rangle\rangle, \| 113-0\rangle\rangle, \| 113-1\rangle\rangle)$ are clearly seen in the plots and they definitely overlap on the symmetric Jain states $|1 ;+\rangle,|2 ; a\rangle,|2 ; b+\rangle$ and $|2 ; c+\rangle$. According to the $W_{1+\infty}$ minimal conformal theory, the interpretation of this branch as the $\nu=2 / 5$ hierarchical Hall state requires the identification of a third edge excitation with $\Delta J=2$. This can reasonably be $\| 113-3\rangle\rangle$ : its density profile is a non-infinitesimal, but in-phase, deformation of the bottom state, and its projection is large on the symmetric Jain states (see Table $(\mathbb{X})$ ). We conclude that the $W_{1+\infty}$ minimal theory consistently describes this branch as the $\nu=2 / 5$ hierarchical state: this is the third definite evidence for this theory.

The next branch $J=108$, i.e. [7,2,1], is plotted in Fig.(15): the profile of the bottom state grows in the bulk and this fits the natural expectation that this branch is a quasi-particle over the $\nu=2 / 5$ state. The families of low-lying states are actually very similar to those of the analogous $N=8$ branch $(J=63)$ in Fig.(7). The multiplicities of the edge excitations should again be $(1,1,3)$ as for the $\nu=2 / 5$ ground state (they are observed in the corresponding $N=8$ branch); instead the actual computing yields $(1,1,2)$ - presumably, the missing state is higher in energy than $\| 110-5\rangle\rangle$.

Let us now discuss the $J=103$ branch, which is drawn in Fig.(16); the corresponding overlaps with the Jain branch $[6,3,1]$ are reported in Table (XI). The density profile of the bottom state is similar to that of the $J=111$ state, i.e. the $\nu=2 / 5$ Hall state; thus, $\| 103-0\rangle\rangle$ can be interpreted as the $\nu=3 / 7$ hierarchical state for $N=10$. The overlap matrix is rather standard for a three-level Jain branch and identifies the CFT labels of the $\Delta J=1$ low-lying states; on the other hand, the $\Delta J=2$ excitations cannot be analysed for the usual reason that there is a single electron in the highest effective Landau level.

The edge excitations are identified by the density profiles and their multiplicities are found to be $(1,2,4)$ : this is in disagreement with the $c=3 W_{1+\infty}$ minimal conformal theory, which predicts the values $(1,1,3)$ (see Table (III)). It is possible that there is an accidental degeneracy with another family $(0,1,1-2)$ of excitations. This interpretation is supported by the analysis of the next branch $J=101$, which is shown in Fig. (17). The bottom state is identified as a quasi-particle over the $\nu=3 / 7$ state, as expected; the multiplicities $(1,1, \ldots)$ of its edge excitations are again in agreement with the $W_{1+\infty}$ minimal theory.

In conclusion, the $N=10$ spectrum presents the general features already encountered for $N=8$ and 6 ; the edge excitations of its hierarchical states can be interpreted within the $W_{1+\infty}$ minimal conformal theory (and the Laughlin quasi-particles by the one-component Abelian theory, of course). However, we should remark that the finite-size effects are not smaller than in the $N=8$ spectrum; this is contrary to the expectation of a smooth thermodynamic limit towards the conformal field theory.

\section{CONCLUSIONS}

In this paper we have presented a comprehensive analysis of the low-lying spectrum of the electrons in the quantum Hall effect, in the regime of hierarchical Hall states $\nu \leq 2 / 5$. We have gone beyond previous works along these lines: regarding the studies of the composite-fermion correspondence [23] [4], we have done the first detailed analysis of the edge state structure, which was overlooked by the studies on the spherical geometry [7] [8]. We have shown that the Jain composite-fermion theory describes very well the low-lying spectrum; but we also revealed the dynamical mechanism which takes place when two Jain states are allowed: the electrons tend to "pile up", if they are let to freely fill the effective Landau levels.

Moreover, we improved and reviewed previous analyses on the disk geometry [9] [23] [24]. We introduced two new criteria for the analysis of the exact states: i) the plot of their density profiles for distinguishing quasi-particle excitations from new incompressible ground states, and for identifying the real edge excitations within the low-lying states; ii) the interpretation of their overlaps with the Jain states in the language of conformal field theory, with concrete relations among the states and projections thereof.

We have presented a consistent analysis of the low-lying spectrum. We have shown that the edge excitations form specific subset of the low-lying states, by applying the previous criteria and by checking that the Laughlin quasiparticles are described by the well-understood one-component Abelian conformal theory. While all the low-lying states are nicely described by the Jain theory, i.e. by the multi-component Abelian conformal theory [9] [13] [17], the real edge excitations of the hierarchical Hall states match the predictions of the $W_{1+\infty}$ minimal theory [18] (and those of the Laughlin quasi-particles naturally match the one-component Abelian theory). Although the numerical data show some blurs and some finite-size limitations, the general picture seems firmly established; we found four neat positive evidences out of the six hierarchical states with $N=8$ and 10 electrons. In conclusion, we hope that this work will stimulate further analyses of the hierarchical Hall states. 


\section{Acknowledgements}

A. C. and G. R. Z. would like to thank the C.E.R.N. Theory Division and the theory group at L.A.P.P., Annecy, for hospitality. A. C. also thanks the Theory Group of the Centro Atómico Bariloche for hospitality and acknowledges the partial support of the European Community Program FMRX-CT96-0012. G. R. Z. is grateful to I.N.F.N. Sezione di Firenze for hospitality. The work of G. R. Z. is supported by a grant of the Antorchas Foundation (Argentina).

[1] For a review see: R. A. Prange, S. M. Girvin, The Quantum Hall Effect, Springer Verlag, New York (1990).

[2] For a review see: S. Das Sarma and A. Pinczuk, Perspectives in Quantum Hall Effects, Wiley, New York (1996).

[3] R. B. Laughlin, Phys. Rev. Lett. 50 (1983) 1395; for a review see: R. B. Laughlin, Elementary Theory: the Incompressible Quantum Fluid, in [⿰].

[4] J. K. Jain, Phys. Rev. Lett. 63 (1989) 199; Phys. Rev. B 41 (1990) 7653; for reviews see: J. K. Jain, Adv. in Phys. 41 (1992) 105, and Composite Fermions, in [2].

[5] For a review see: H. L. Stormer and D. C. Tsui, Composite Fermions in the Fractional Quantum Hall Effect, in [2]

[6] For a review see: E. Fradkin and A. Lopez, Nucl. Phys. B (Proc. Suppl.) 33C (1993) 67.

[7] X. G. Wu and J. K. Jain, Phys. Rev. B 51 (1995) 1752.

[8] J. K. Jain and R. K. Kamilla, Int. J. Mod. Phys. B 11 (1997) 2621.

[9] For a review, see: X. G. Wen, Int. J. Mod. Phys. 6 B (1992) 1711, Adv. in Phys. 44 (1995) 405.

[10] R. C. Ashoori, H. L. Stormer, L. N. Pfeiffer, K. W. Baldwin and K. West, Phys. Rev. B 45 (1992) 3894;

[11] F. P. Milliken, C. P. Umbach and R. A. Webb, Solid State Commun. 97 (1996) 309; P. Fendley, A. W. W. Ludwig and H. Saleur, Phys. Rev. B 52 (1995) 8934; for a review, see: C. L. Kane and M. P. A. Fisher, Edge-State Transport, in [2].

[12] V. J. Goldman and B. Su, Science 267 (1995) 1010; R. de-Picciotto et al., cond-mat/9707289; L. Saminadayar et al., cond-mat/9706307.

[13] J. Fröhlich and A. Zee, Nucl. Phys. 364 B (1991) 517; X.-G. Wen and A. Zee, Phys. Rev. 46 B (1993) 2290. J. Fröhlich and E. Thiran, J. Stat. Phys. 76 (1994) 209; J. Frölich, T. Kerler, U. M. Studer and E. Thiran, Nucl. Phys. B 453 (1995) 670 .

[14] A. A. Belavin, A. M. Polyakov and A. B. Zamolodchikov, Nucl. Phys. B 241 (1984) 333; for a review see: P. Ginsparg, Applied Conformal Field Theory, in Fields, Strings and Critical Phenomena, Les Houches School 1988, E. Brezin and J. Zinn-Justin eds., North-Holland, Amsterdam (1990).

[15] M. Stone, Ann. Phys. (NY) 207 (1991) 38.

[16] A. Cappelli, G. V. Dunne, C. A. Trugenberger and G. R. Zemba, Nucl. Phys. 398 B (1993) 531.

[17] N. Read, Phys. Rev. Lett. 65 (1990) 1502.

[18] A. Cappelli, C. A. Trugenberger and G. R. Zemba, Nucl. Phys. 448 (1995) 470; for a review, see: Nucl. Phys. (Proc. Suppl.) B 45A (1996) 112.

[19] S. Iso, D. Karabali and B. Sakita, Nucl. Phys. B 388 (1992) 700, Phys. Lett. B 296 (1992) 143.

[20] A. Cappelli, C. A. Trugenberger and G. R. Zemba, Nucl. Phys. 396 B (1993) 465, Phys. Lett. 306 B (1993) 100; for a review, see: A.Cappelli, G.V.Dunne, C.A.Trugenberger and G.R.Zemba, Nucl. Phys. B (Proc. Suppl.) 33C (1993) 21.

[21] A. Cappelli, C. A. Trugenberger and G. R. Zemba, Phys. Rev. Lett. 72 (1994) 1902.

[22] F. D. M. Haldane, The Hierarchy of Fractional States and Numerical Studies, in 11.

[23] G. Dev and J. K. Jain, Phys. Rev. 45 B (1992) 1223.

[24] M. Kasner and W. Apel, Phys. Rev. 48 B (1993) 11435; Ann. Physik 3 (1994) 433.

[25] A. Cappelli and G. R. Zemba, Hamiltonian Formulation for the Minimal Models of the Incompressible Quantum Hall Fluids, to appear.

[26] V. Kac and A. Radul, Comm. Math. Phys. 157 (1993) 429; H. Awata, M. Fukuma, Y. Matsuo and S. Odake, Prog. Theor. Phys. (Supp.) 118 (1995) 343; E. Frenkel, V. Kac, A. Radul and W. Wang, Comm. Math. Phys. 170 (1995) 337.

[27] A. M. Polyakov, Int. J. Mod. Phys. A 5 (1990) 833; M. Bershadsky and H. Ooguri, Comm. Math. Phys. 126 (1989) 49.

[28] F. D. M. Haldane Phys. Rev. Lett. 51 (1983) 605; B. I. Halperin, Phys. Rev. Lett. 52 91984) 1583; M. Greiter, Phys. Lett. B 336 (1994) 48. 
TABLE I. Bottom states of the Jain theory for $N=8$, ordered by the decreasing angular momentum $J \geq 63$, and the corresponding approximated energies $E^{(0)}$, in units of $E_{D}$.

\begin{tabular}{|c|r|r|}
\hline \hline \hline$N=8$ Jain states & $J$ & $E^{(0)}$ \\
\hline$[8]$ & 84 & 0 \\
{$[7,1]$} & 76 & 1 \\
{$[6,2]$} & 70 & 2 \\
\hline$[6,1,1]$ & 68 & 3 \\
{$[5,3]$} & 66 & 3 \\
\hline$[4,4]$ & 64 & 4 \\
{$[5,2,1]$} & 63 & 4 \\
\hline \hline
\end{tabular}

TABLE II. The number of edge excitations for the Laughlin Hall fluid $\nu=1 / 3$ and its quasi-particles (first row); for the hierarchical fluids $\nu=2 / 5$ (second and third rows) and $\nu=3 / 7$ (forth and fifth rows), according to the two relevant conformal field theories with central charge $c$.

\begin{tabular}{|c|l|lll|}
\hline \hline \hline$c$ & $\Delta J$ & 0 & 1 & 2 \\
\hline 1 & One-component Abelian & 1 & 1 & 2 \\
\hline 2 & Two-component Abelian & 1 & 2 & 3 \\
& Minimal Incompressible & 1 & 1 & 3 \\
\hline 3 & Three-component Abelian & 1 & 3 & 3 \\
& Minimal Incompressible & 1 & 1 & 3 \\
\hline \hline
\end{tabular}

TABLE III. Overlap matrices for a Laughlin quasi-particle branch. The $N=8$ low-lying exact states are denoted by $\| 70-i\rangle\rangle$, $i=0,1, \| 71-j\rangle\rangle, j=0,1$, and $\| 72-k\rangle\rangle, k=0, \ldots, 4$. The corresponding orthogonalized Jain states are $|[6,2]\rangle,|1 ; \pm\rangle$ at $\Delta J=1$, and $(|2 ; a\rangle,|2 ; b \pm\rangle,|2 ; c \pm\rangle)$ at $\Delta J=2$.

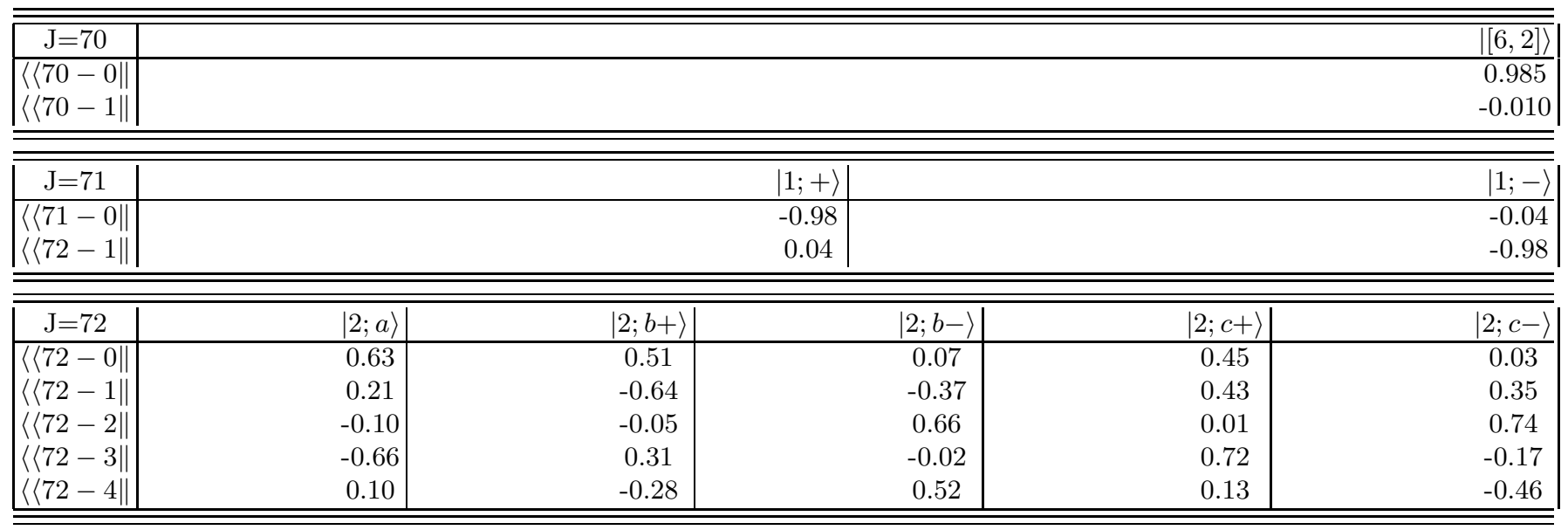


TABLE IV. Overlap matrices for the $\nu=2 / 5$ branch with $N=8$. The exact states are $\| 66-i\rangle\rangle, i=0,1, \| 67-j\rangle\rangle$, $j=0,1,2$, and $\| 68-k\rangle\rangle, k=0, \ldots, 5$; the orthogonalized Jain states are $|[5,3]\rangle,|1 ; \pm\rangle$ and $(|2 ; a\rangle,|2 ; b \pm\rangle,|2 ; c \pm\rangle)$.

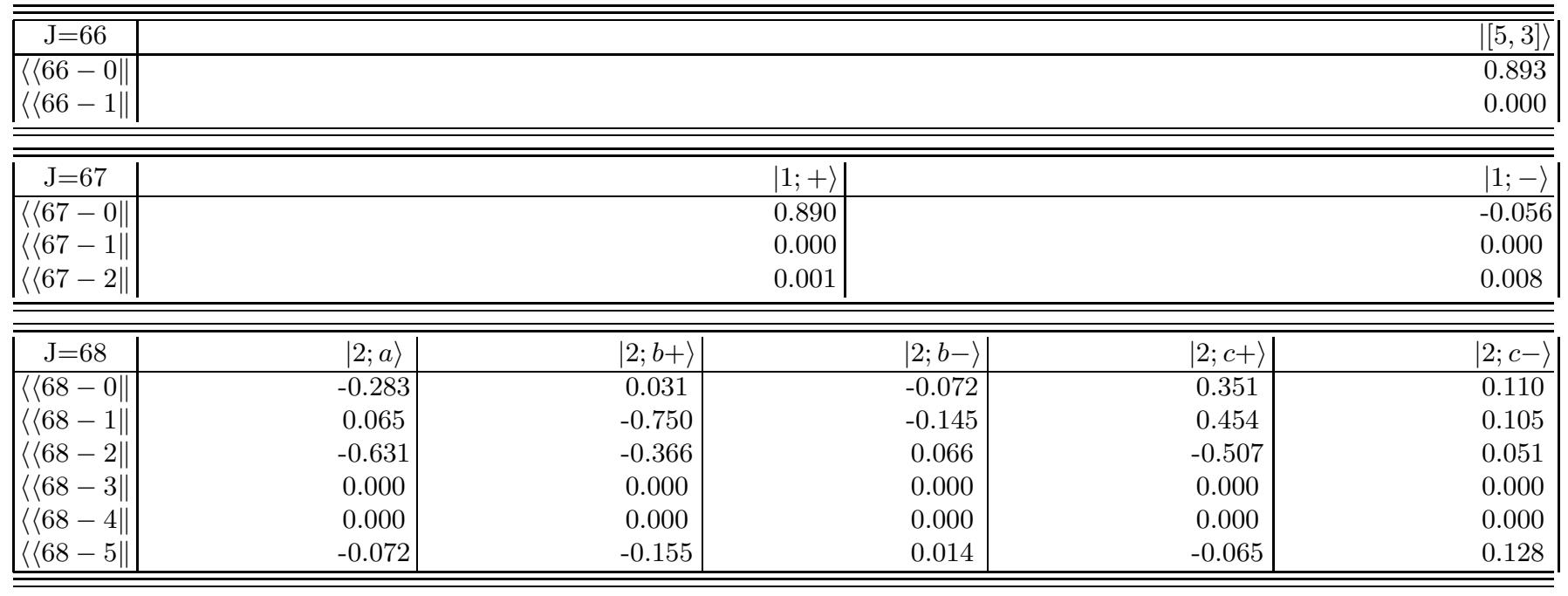

TABLE V. Overlap matrices of the quasi-particle branch over the $\nu=2 / 5$ state for $N=8$. The exact states are $\| 63-i\rangle\rangle$, $i=0,1$, and $\| 64-j\rangle\rangle, j=0,1,2$; the orthogonalized Jain states are $|[5,2,1]\rangle$ and its excitations $|1 ; x\rangle, x=a, b, c$.

\begin{tabular}{|c|c|c|c|}
\hline $\mathrm{J}=63$ & & & $\overline{|[5,2,1]\rangle}$ \\
\hline$\langle\langle 63-0 \|$ & & & -0.959 \\
\hline$\langle\langle 63-1 \|$ & & & 0.000 \\
\hline $\mathrm{J}=64$ & $|1 ; a\rangle$ & $|1 ; b\rangle$ & $|1 ; c\rangle$ \\
\hline$\langle\langle 64-0 \|$ & 0.94 & 0.01 & $\overline{0.14}$ \\
\hline$\langle\langle 64-1 \|$ & 0.06 & -0.90 & -0.32 \\
\hline
\end{tabular}

TABLE VI. Jain bottom states for $N=6$, ordered by the decreasing angular momentum $J \geq 33$, and the corresponding energies $E^{(0)}$, in units of $E_{D}$.

\begin{tabular}{|c|r|r|}
\hline \hline \hline$N=6$ Jain states & $J$ & $E^{(0)}$ \\
\hline$[6]$ & 45 & 0 \\
{$[5,1]$} & 39 & 1 \\
{$[4,2]$} & 35 & 2 \\
\hline$[4,1,1]$ & 33 & 3 \\
{$[3,3]$} & 33 & 3 \\
\hline \hline
\end{tabular}


TABLE VII. Typical values of the average density $\rho(0)$ and of the angular momentum for idealized flat droplets of incompressible fluids.

\begin{tabular}{|c|c|ccc|}
\hline \hline$\nu$ & $2 \pi \rho(0)$ & $N=6$ & $J$ & \\
& & $N=8$ & \\
\\
\hline $1 / 3$ & 0.33 & 45 & 84 & 10 \\
$2 / 5$ & 0.40 & 37.5 & 70 & 135 \\
$3 / 7$ & 0.43 & 35 & 65.3 & 112.5 \\
\hline \hline
\end{tabular}

TABLE VIII. Overlap matrices for the candidate $\nu=3 / 7$ branch with $N=6$. The exact states $\| 33-i\rangle\rangle$, $(i=0,1)$, are compared with the two Jain bottom states $|[3,3]\rangle$ and $|[4,1,1]\rangle ;$ the $\Delta J=1$ low-lying exact states are $\| 34-j\rangle\rangle,(j=0,1,2)$, and the Jain states $|1 ; x\rangle, x=a, b, c$, are excitations of $|[4,1,1]\rangle$.

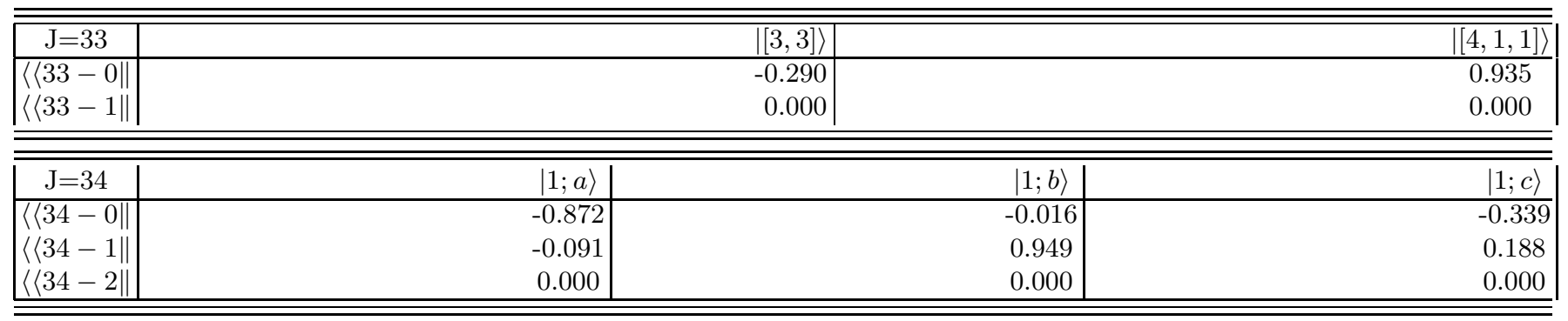

TABLE IX. Jain bottom states for $N=10$ with angular momentum $J \geq 100$, and the corresponding energies $E^{(0)}$, in units of $E_{D}$.

\begin{tabular}{|c|c|c|}
\hline$(N=10)$ Jain state & $J$ & $E^{(0)}$ \\
\hline$[10]$ & 135 & 0 \\
\hline$[9,1]$ & 125 & 1 \\
\hline$[8,2]$ & 117 & 2 \\
\hline$[7,3]$ & 111 & 3 \\
\hline$[7,2,1]$ & 108 & 4 \\
\hline$[6,3,1]$ & 103 & 5 \\
\hline$[7,1,1,1]$ & 105 & 6 \\
\hline$[6,2,2]$ & 101 & 6 \\
\hline$[5,4,1]$ & 100 & 6 \\
\hline
\end{tabular}


TABLE X. Overlap matrices for the $\nu=2 / 5$ branch with $N=10$. The exact states are $\| 111-i\rangle\rangle, i=0,1, \| 112-j\rangle\rangle$, $j=0,1$, and $\| 113-k\rangle\rangle, k=0, \ldots, 4$; the orthogonalized Jain states are $|[7,3]\rangle,|1 ; \pm\rangle$ and $(|2 ; a\rangle,|2 ; b \pm\rangle,|2 ; c \pm\rangle)$.

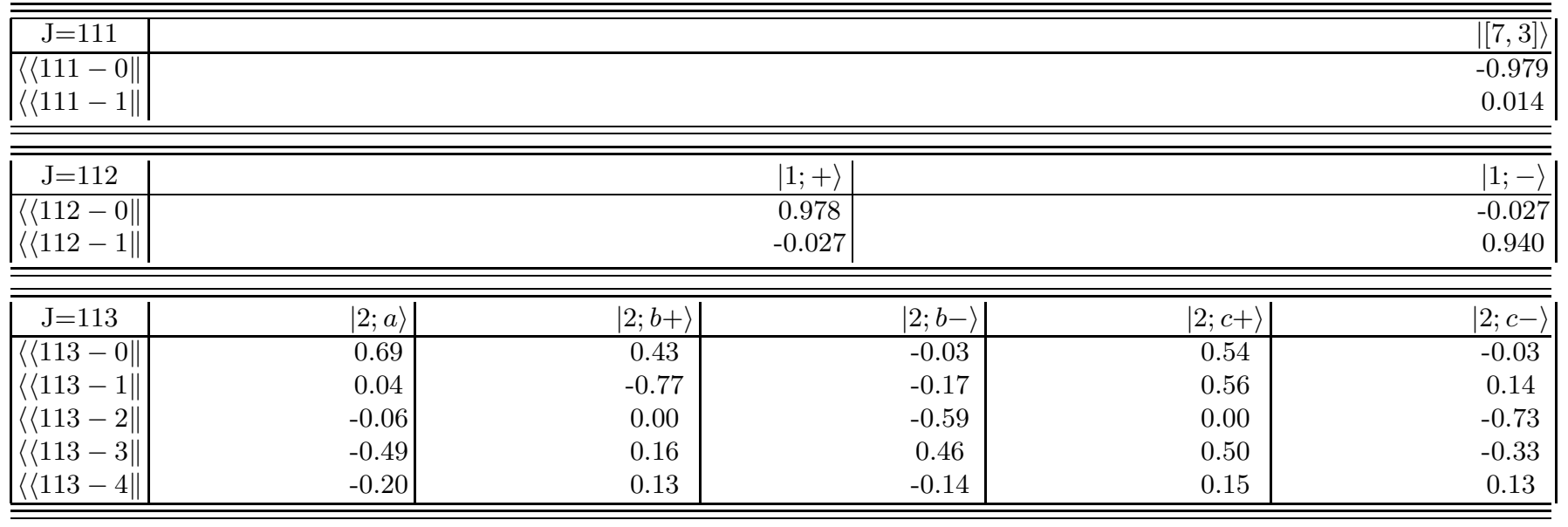

TABLE XI. Overlap matrices for the $\nu=3 / 7$ branch for $N=10$. The exact states are $\| 103-i\rangle\rangle, i=0,1$, and $\| 104-j\rangle\rangle$, $j=0,1,2$; the orthogonalized Jain states are $|[6,3,1]\rangle$ and its excitations $|1 ; x\rangle, x=a, b, c$.

\begin{tabular}{|c|c|c|c|}
\hline $\bar{J} \mathrm{~J}=103$ & & & $\overline{|[6,3,1]\rangle}$ \\
\hline$\langle\langle 103-0 \|$ & & & -0.954 \\
\hline$\langle\langle 103-1 \|$ & & & 0.000 \\
\hline $\mathrm{J}=104$ & $|1 ; a\rangle$ & $|1 ; b\rangle$ & $\overline{|1 ; c\rangle}$ \\
\hline$\langle\langle 104-0 \|$ & -0.947 & 0.030 & -0.105 \\
\hline$\langle\langle 104-1 \|$ & 0.031 & -0.779 & -0.507 \\
\hline
\end{tabular}




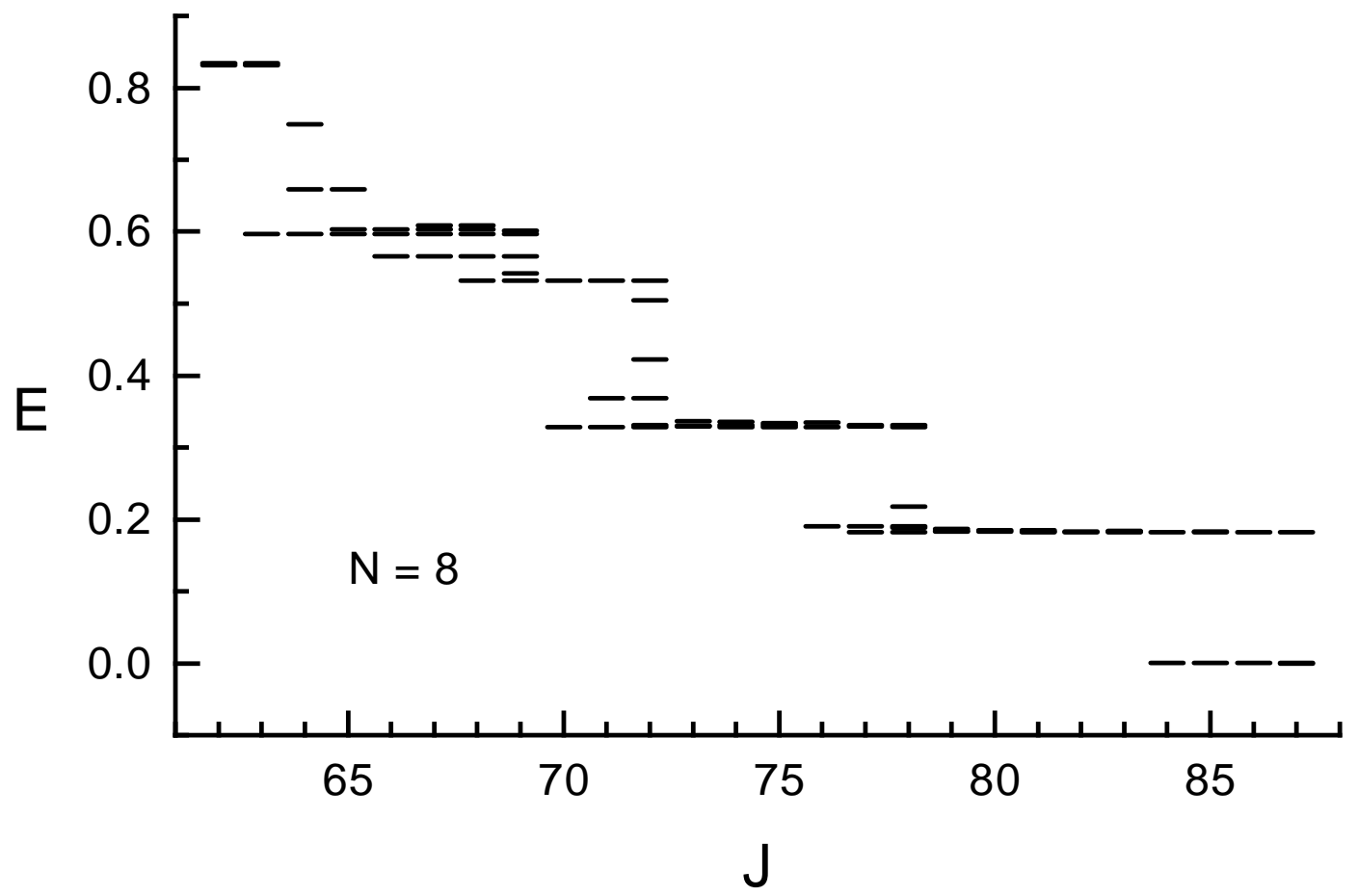

FIG. 1. Exact numerical spectrum for $N=8$ on the disk geometry: only the first few low-lying states are displayed.

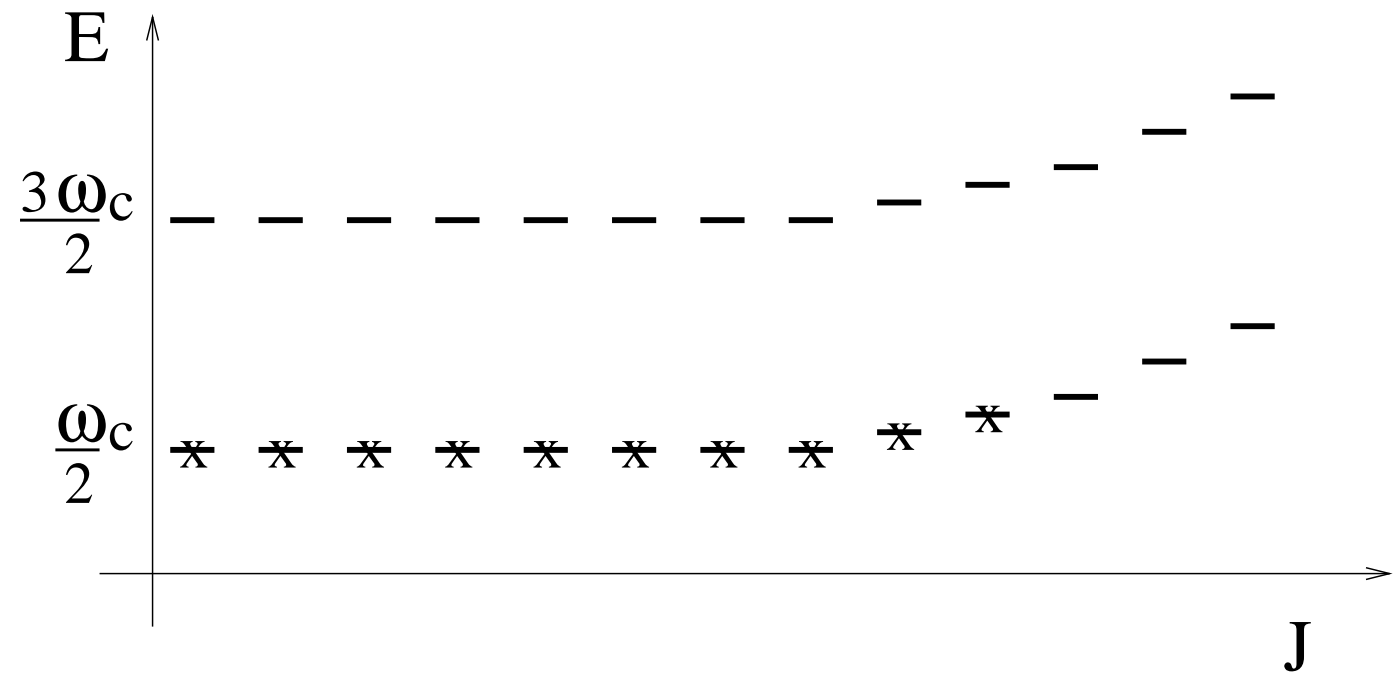

FIG. 2. The Landau levels on a disk: the dashes represent one-particle states, whose energy grows at large $J$ due to the confining potential. The crosses represent the electrons filling the first Landau level. 


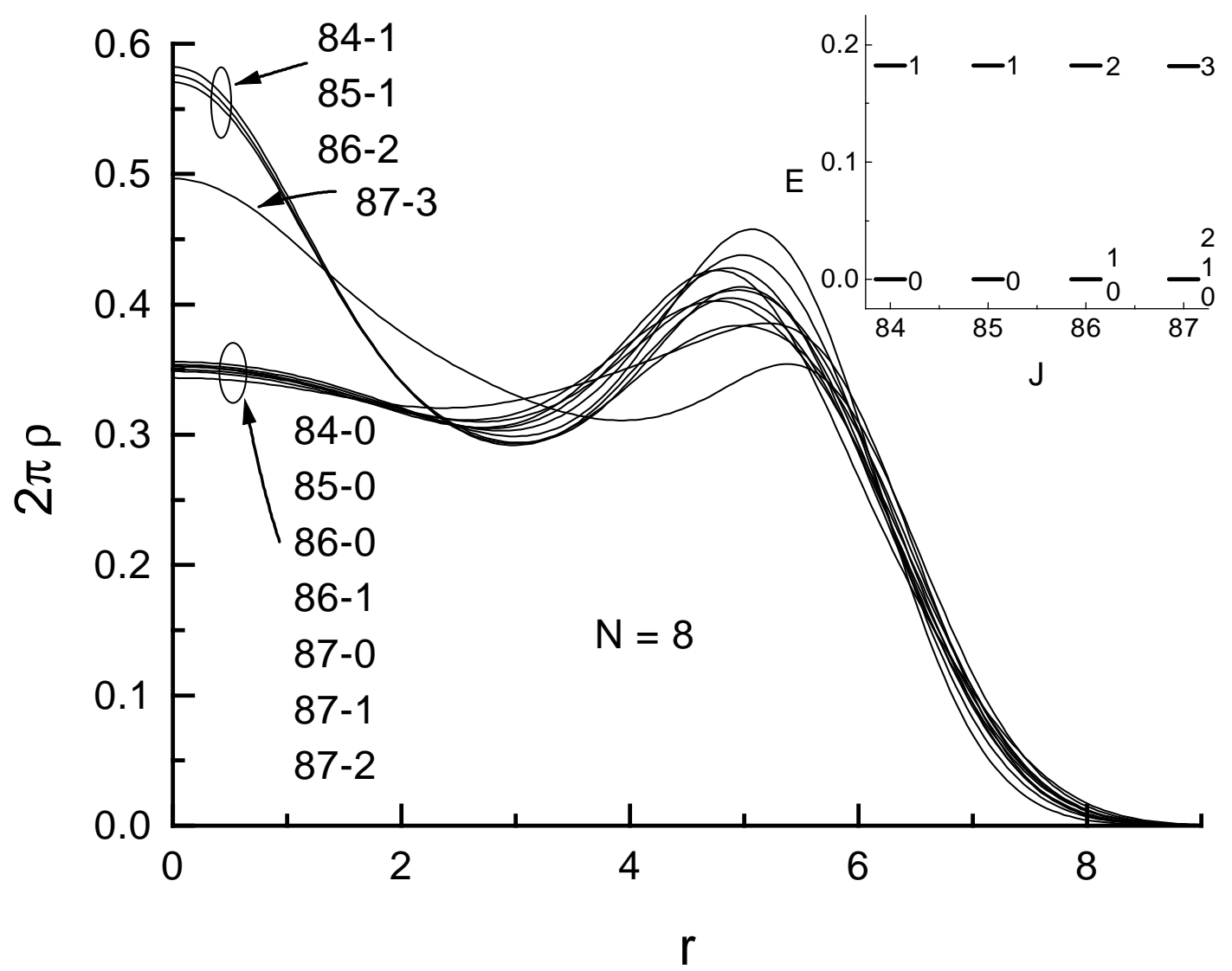

FIG. 3. Density profiles and energies for the branch of the $\nu=1 / 3$ Laughlin ground state.

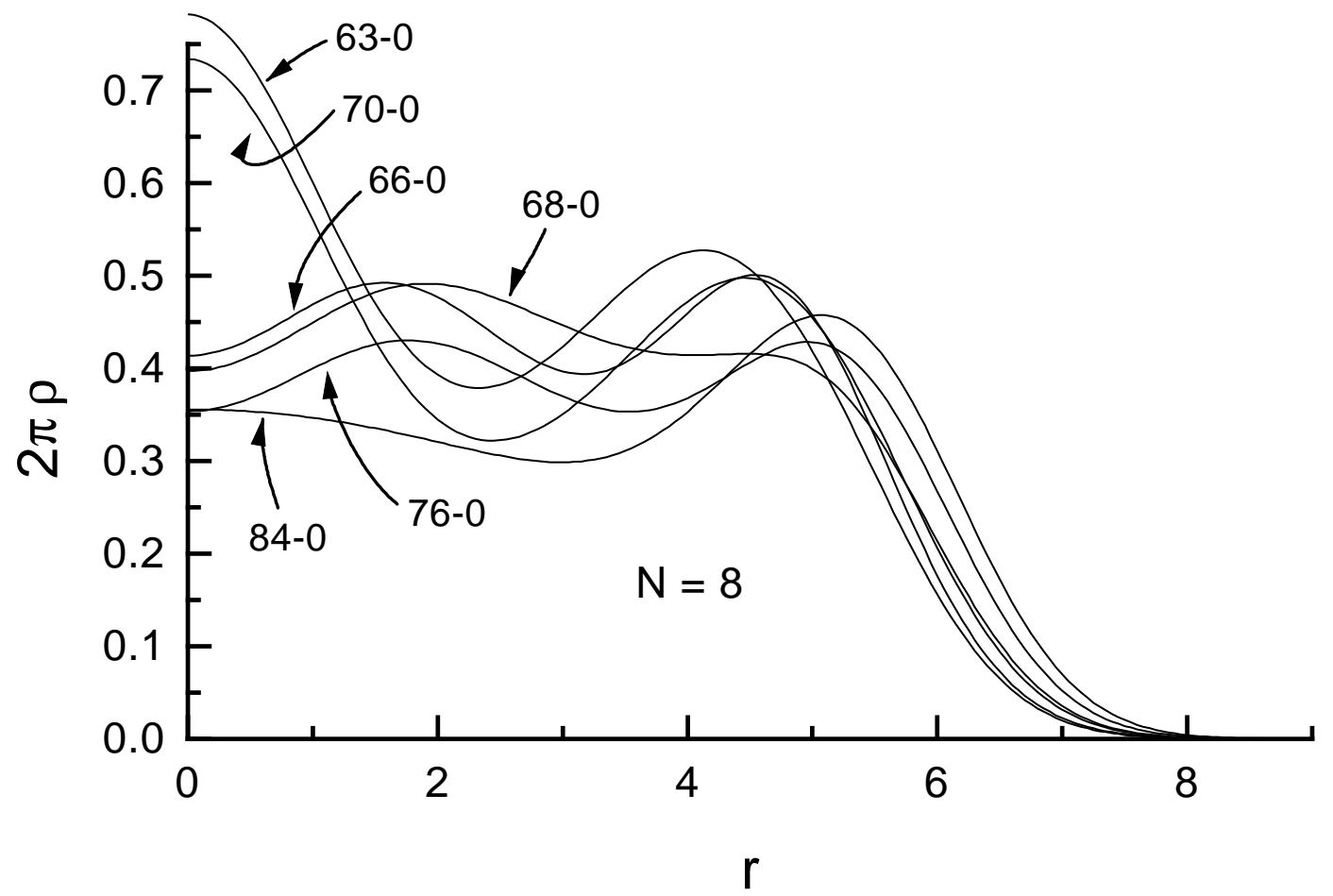

FIG. 4. Comparison of the density profiles for all the $N=8$ bottom states. 


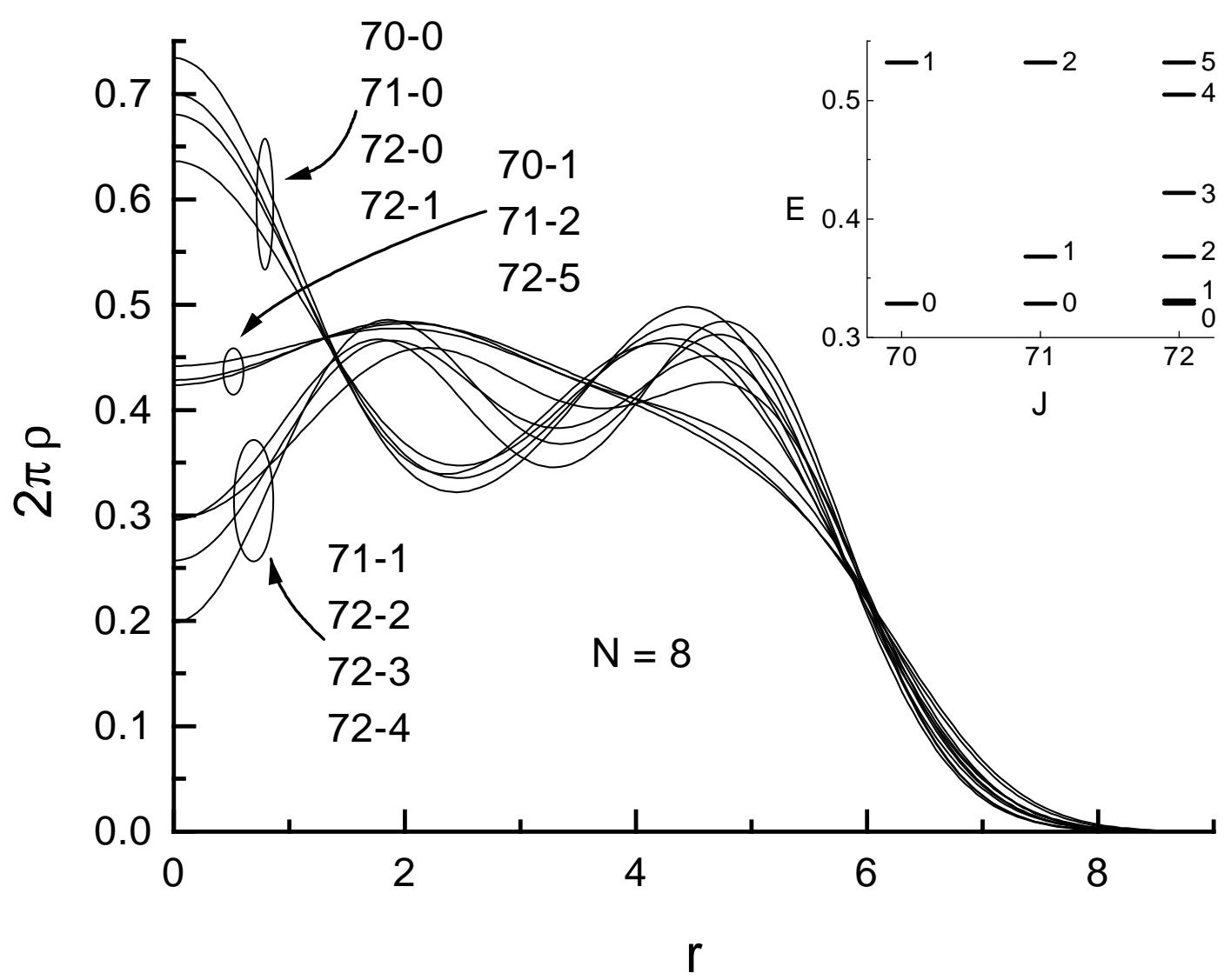

FIG. 5. Density profiles and energies of a quasi-particle branch over the Laughlin state.

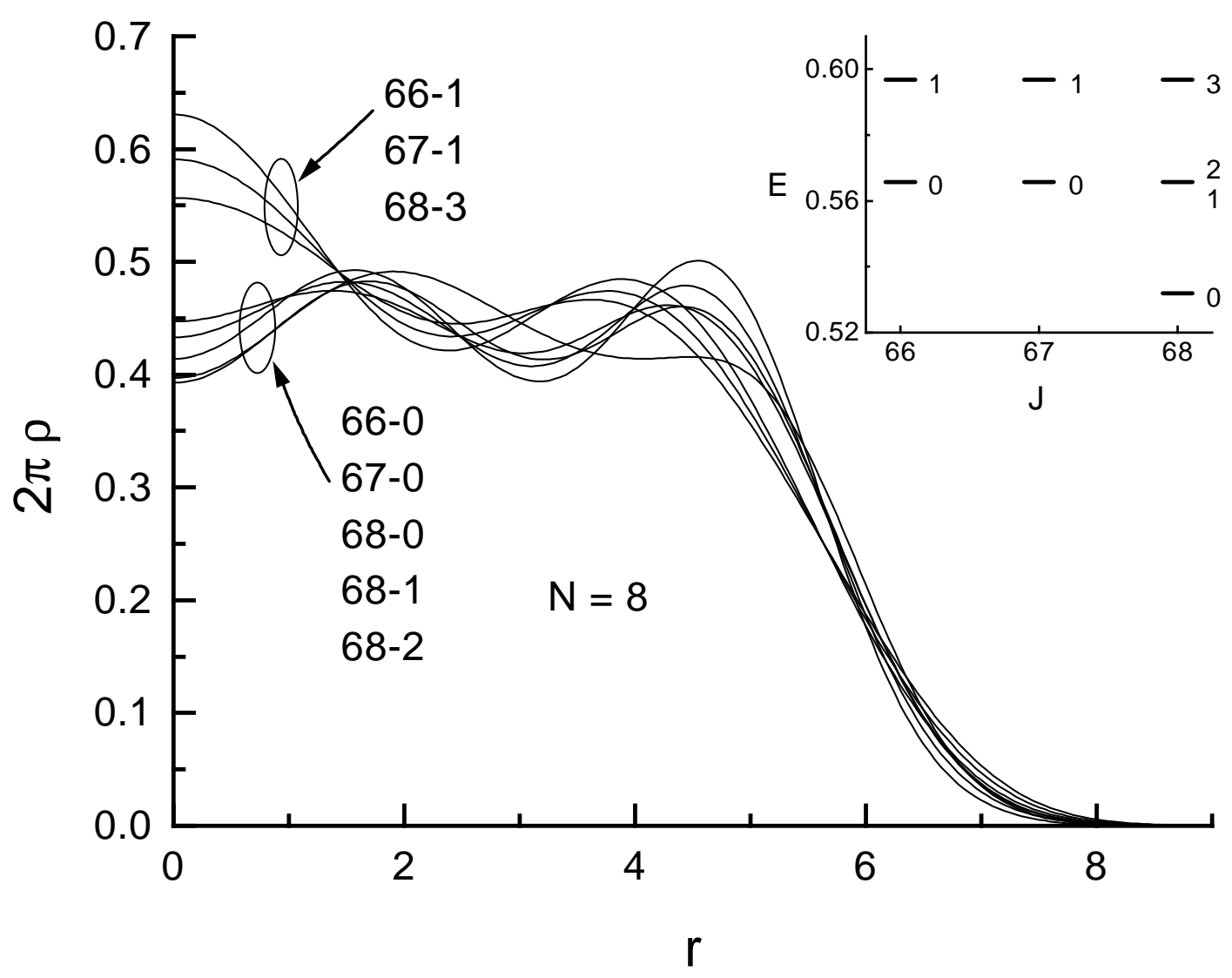


FIG. 6. Density profiles and energies for the branch of the $\nu=2 / 5$ hierarchical ground state.

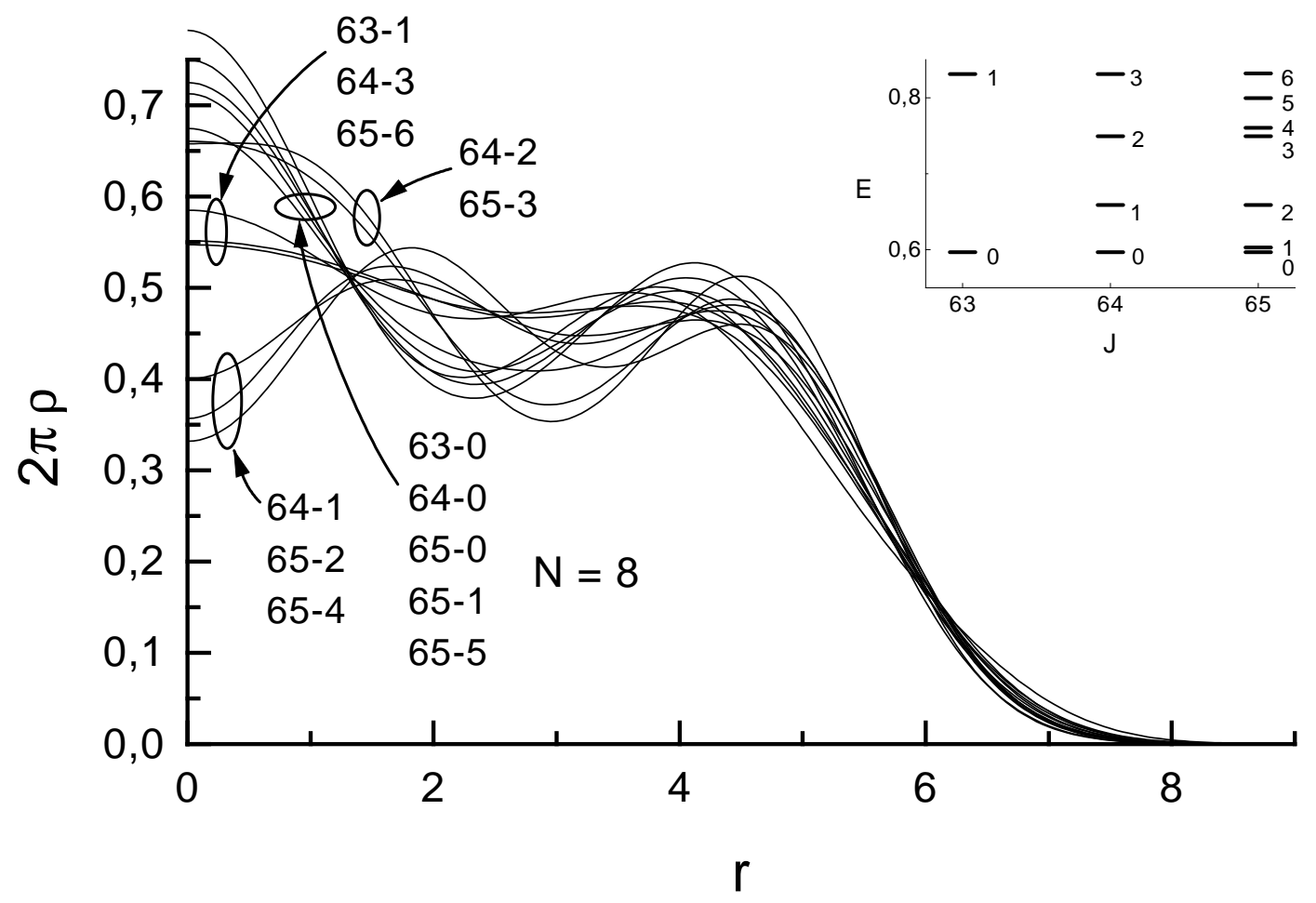

FIG. 7. Density profiles and energies for the quasi-particle branch over the $\nu=2 / 5$ state.

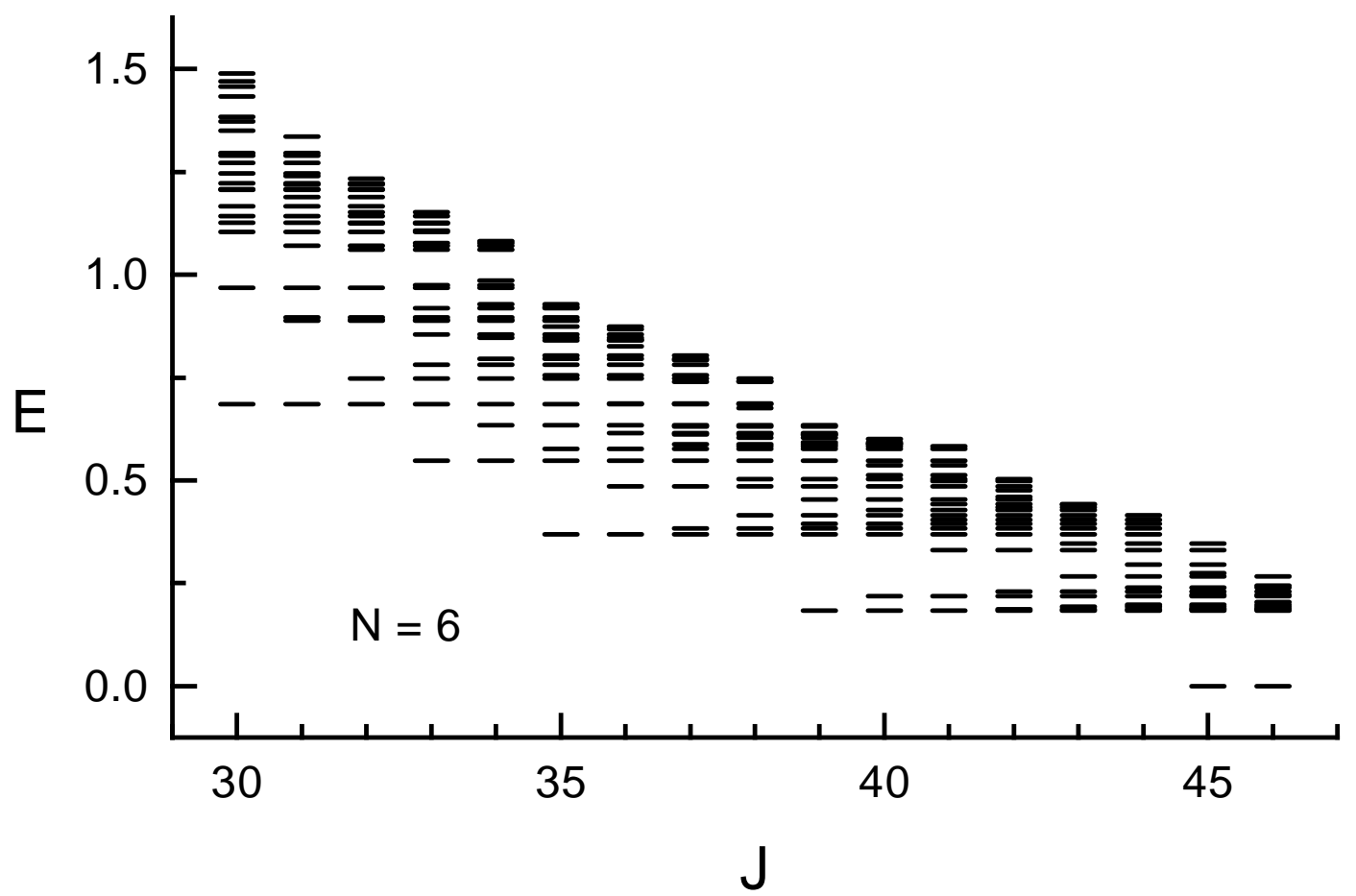

FIG. 8. Exact numerical spectrum for $N=6$. 


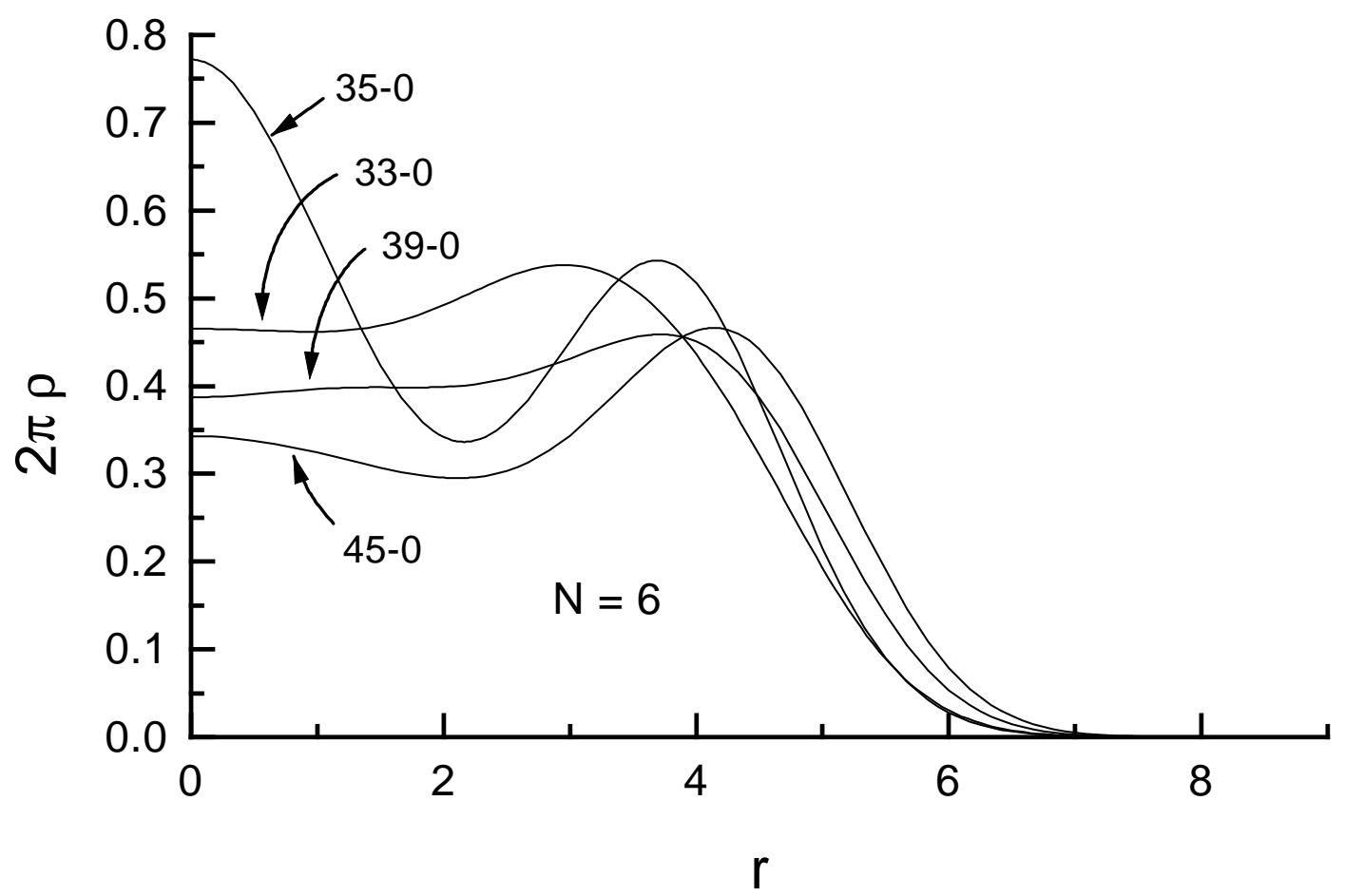

FIG. 9. Comparison of the density profiles for all the $N=6$ bottom states.

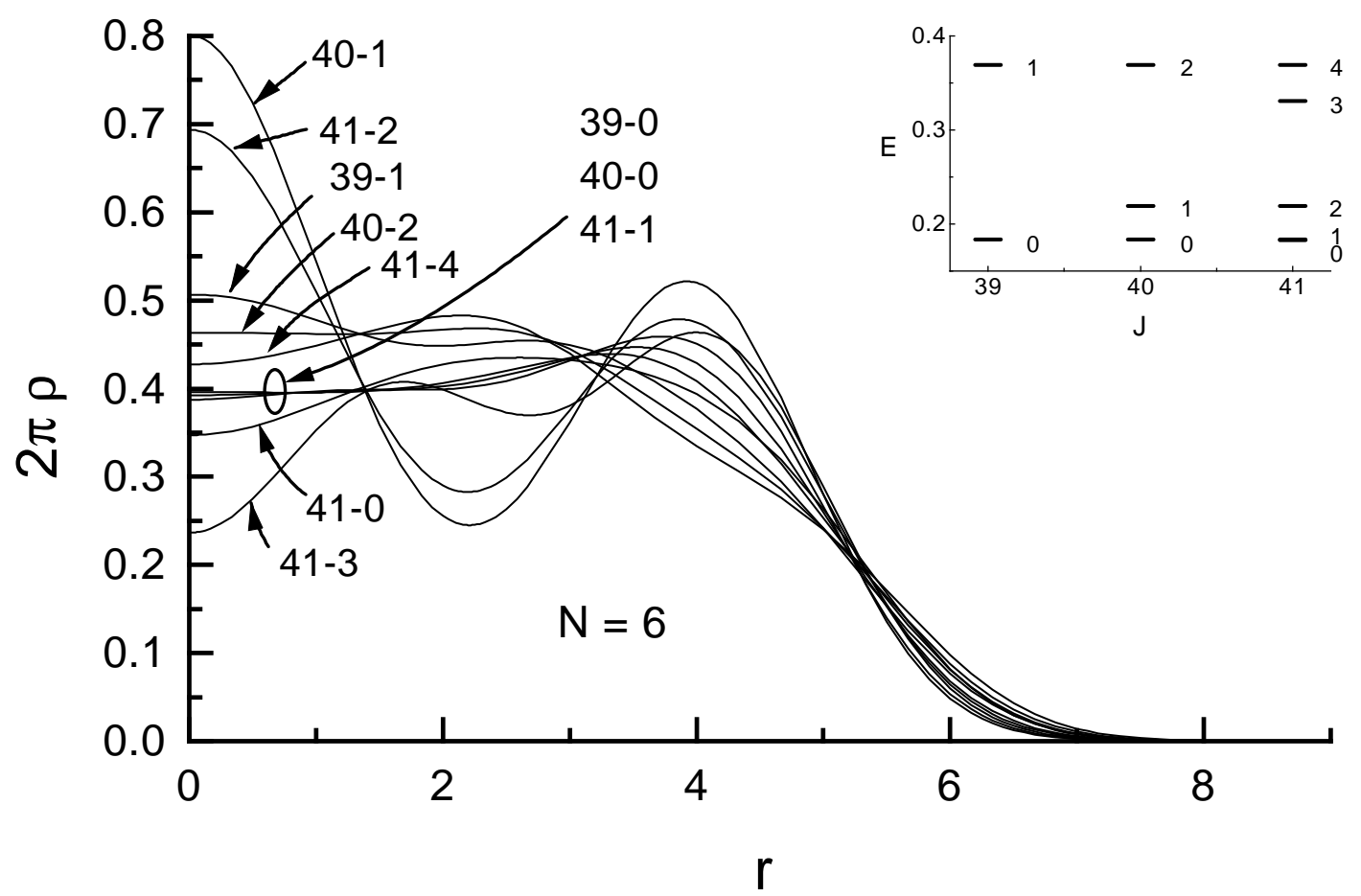

FIG. 10. Density profiles of $N=6$ branch which can be interpreted as the $\nu=2 / 5$ ground state. 


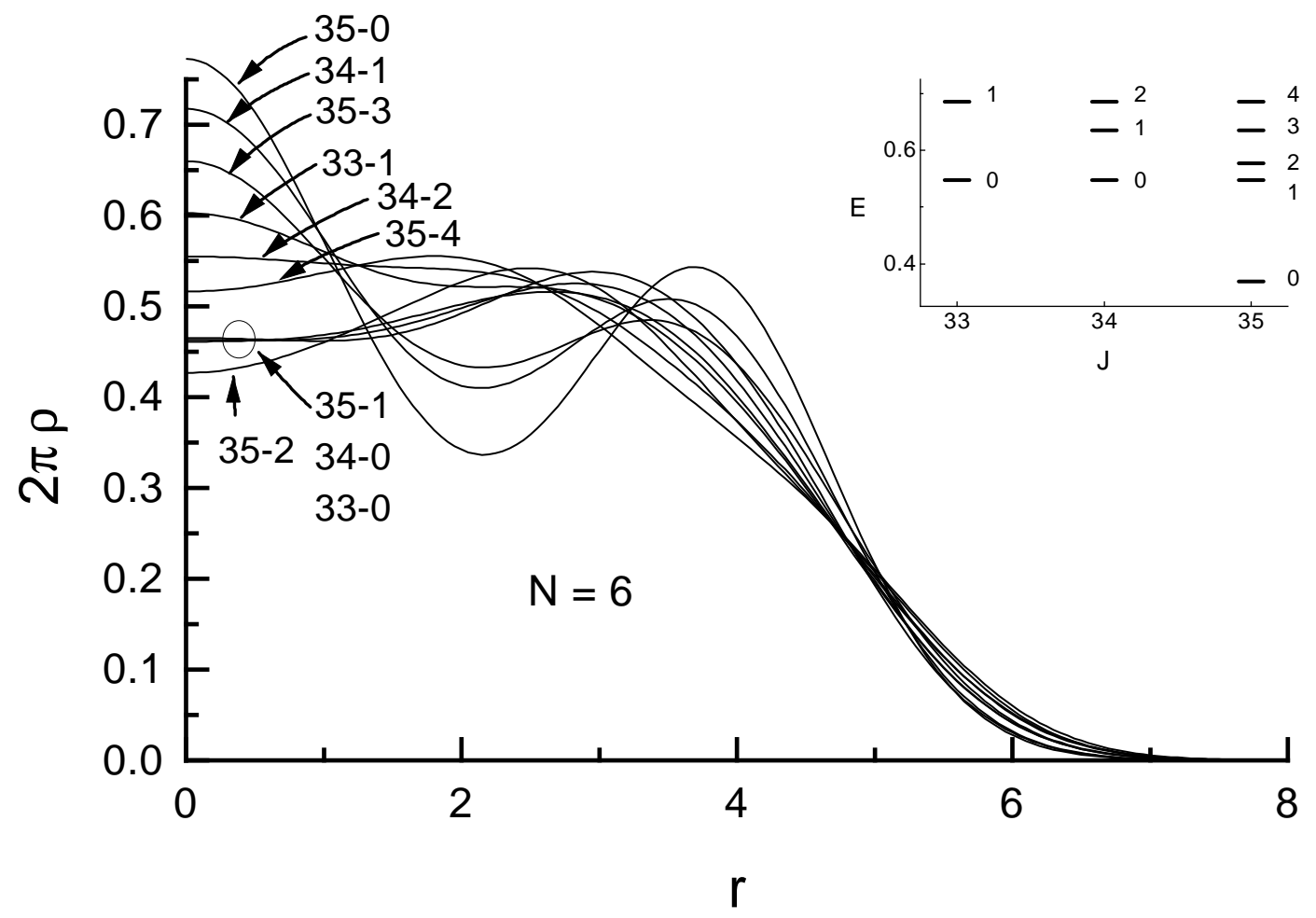

FIG. 11. Density profiles of $N=6$ branch which can be interpreted as the $\nu=3 / 7$ ground state.

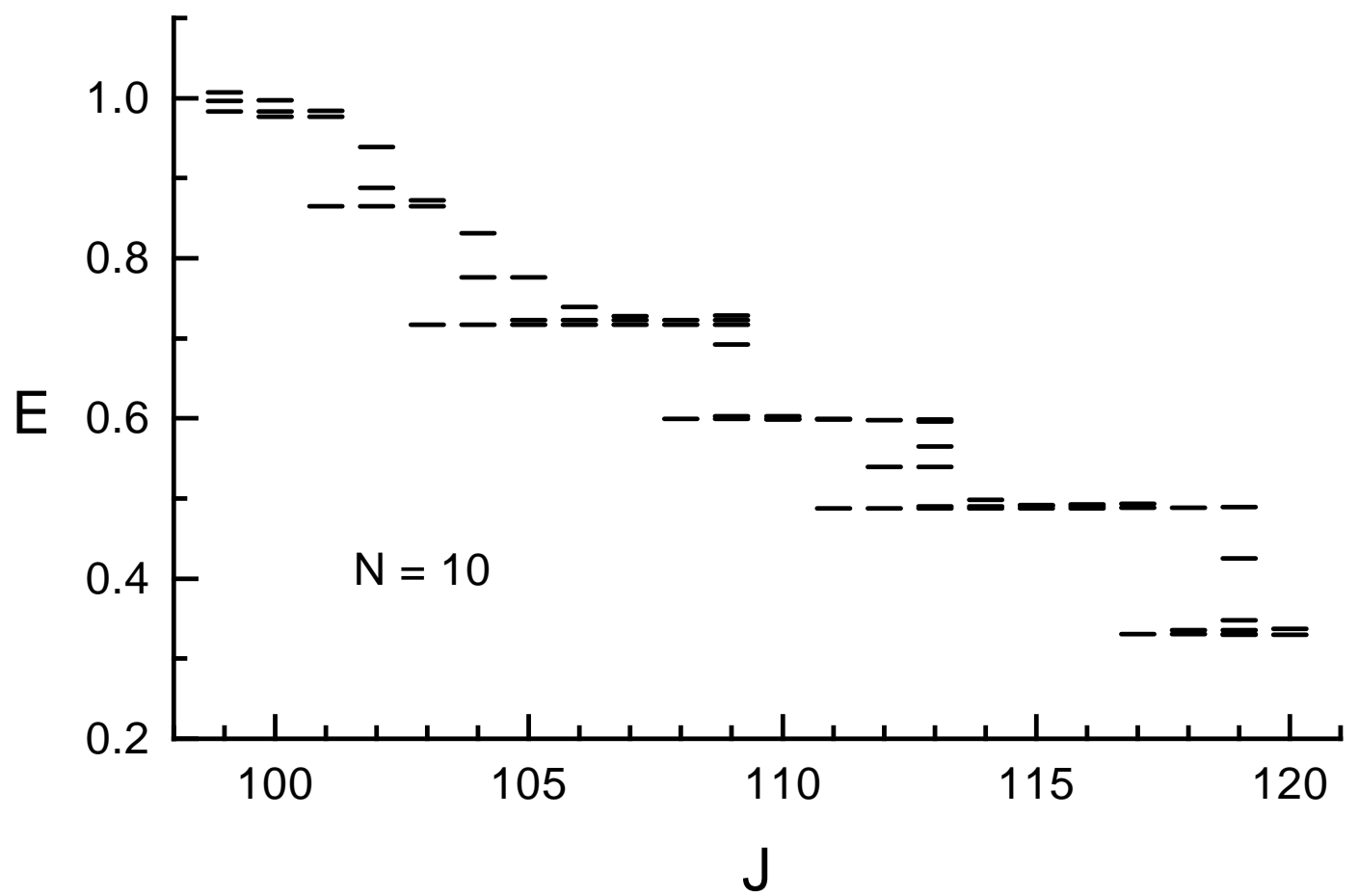

FIG. 12. Exact numerical spectrum for $N=10$ : only the first few low-lying states are displayed. 


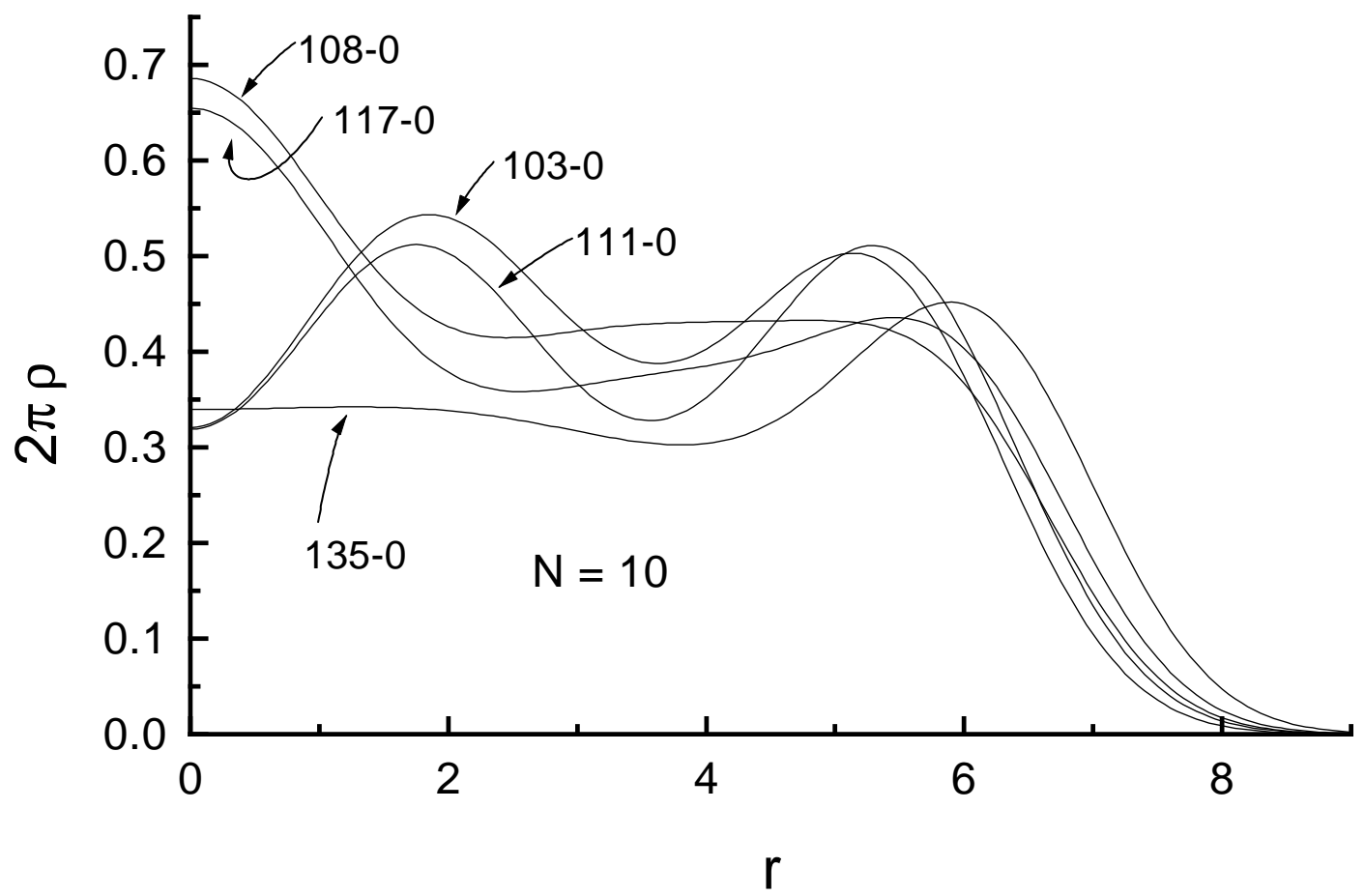

FIG. 13. Comparison of the density profiles for all the $N=10$ bottom states.

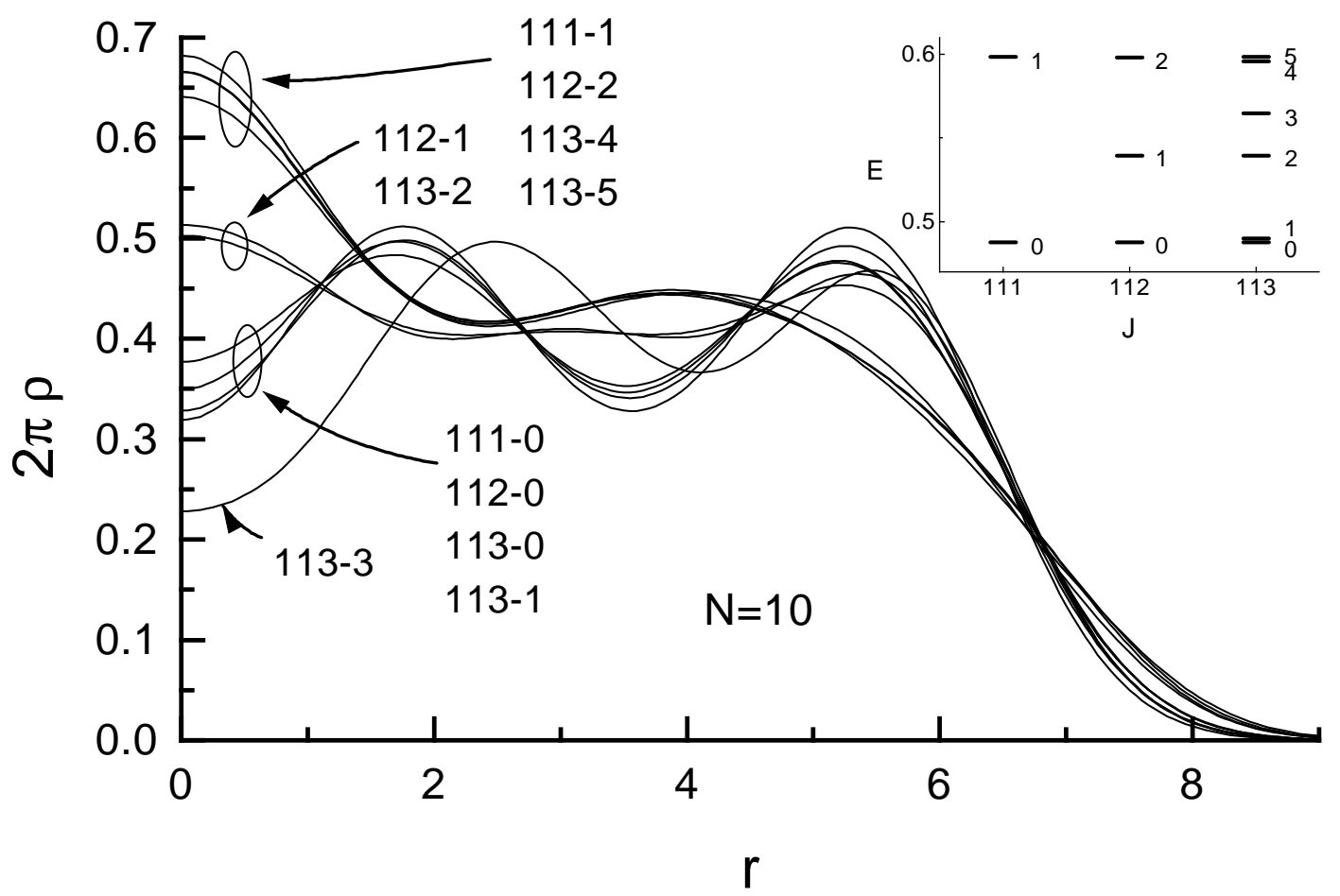

FIG. 14. Density profiles and energies for the branch of the $\nu=2 / 5$ ground state. 


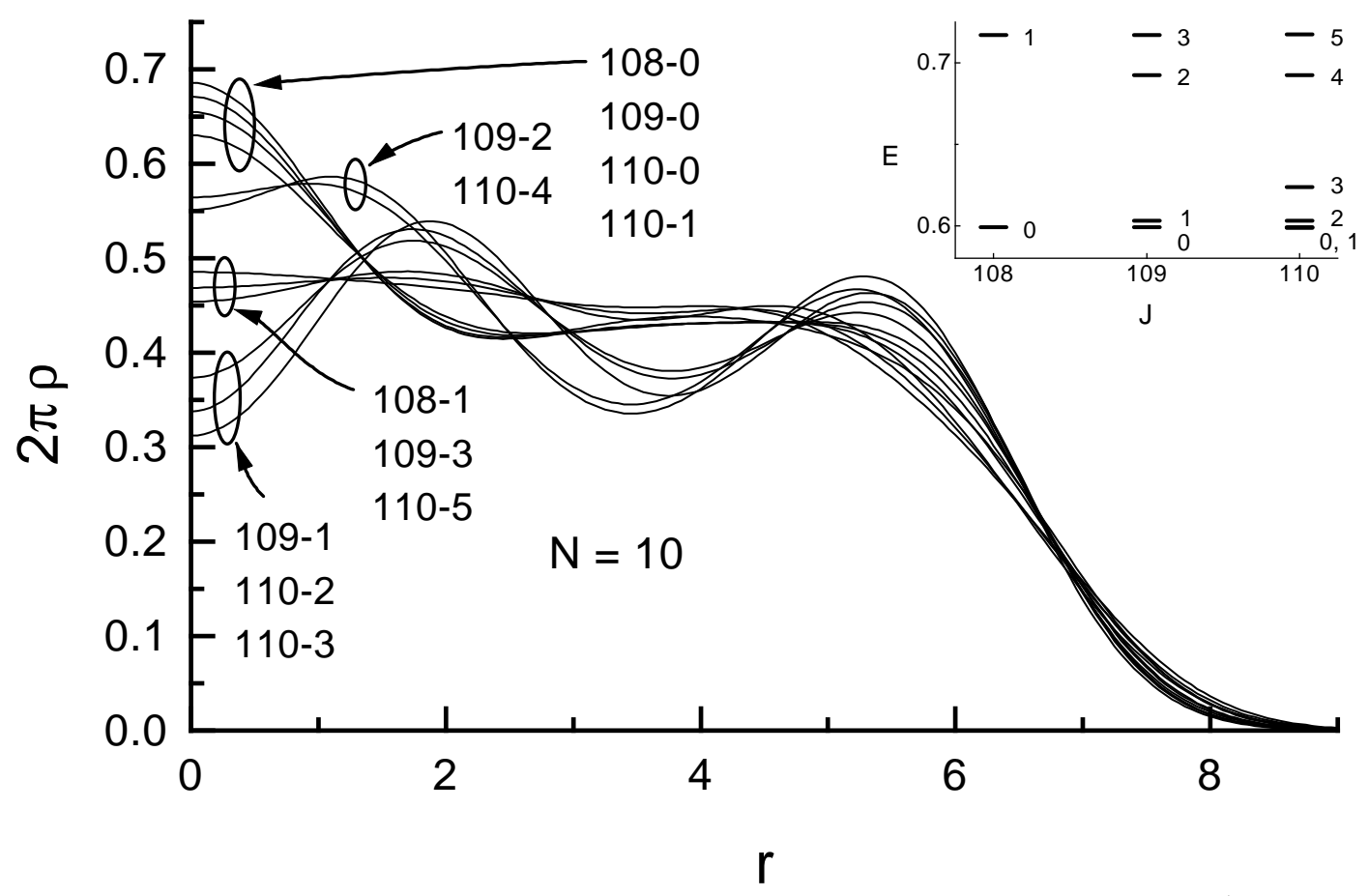

FIG. 15. Density profiles and energies for the branch of a quasi-particle over the $\nu=2 / 5$ state.

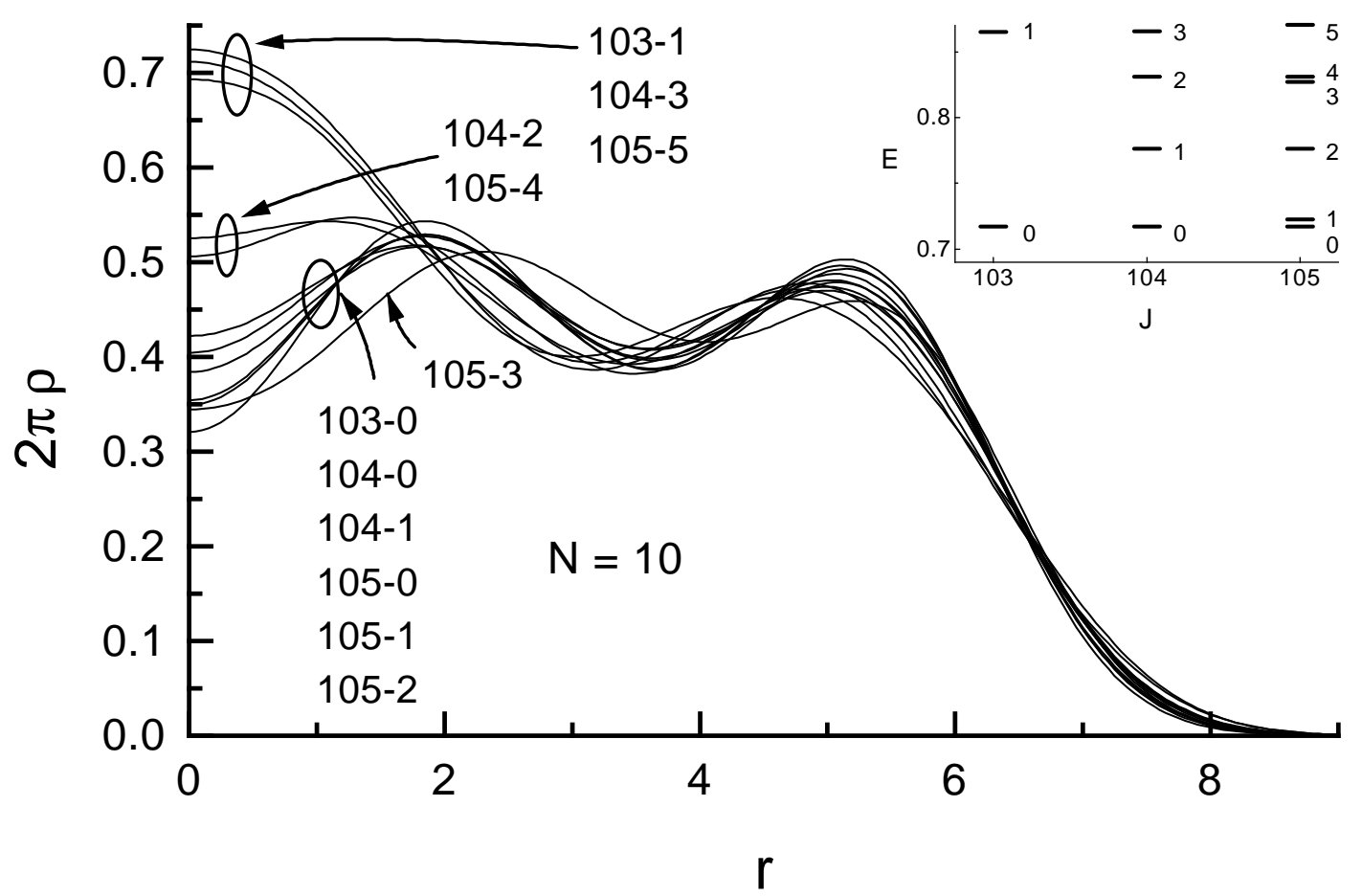

FIG. 16. Density profiles and energies for the branch of the $\nu=3 / 7$ ground state. 


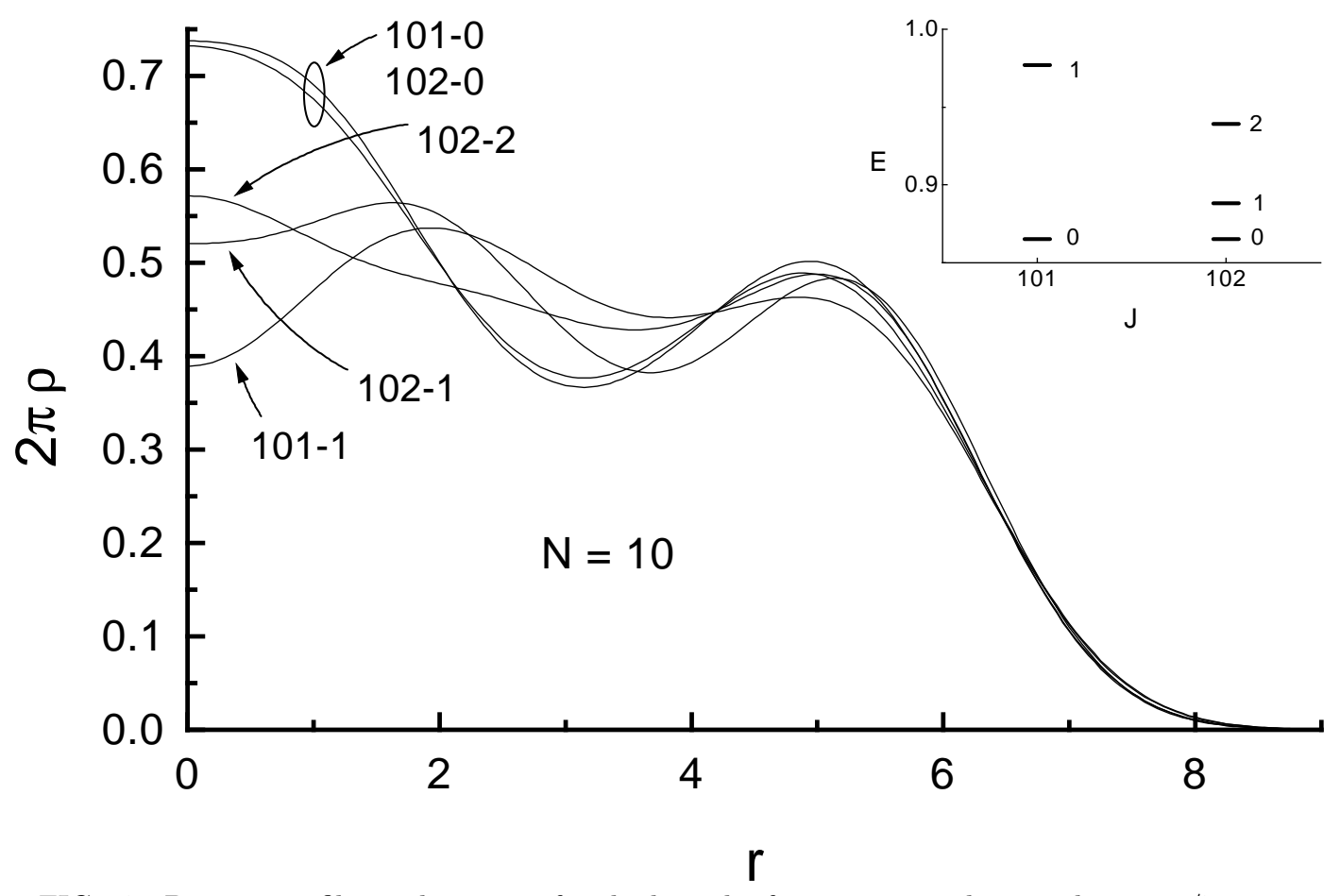

FIG. 17. Density profiles and energies for the branch of a quasi-particle over the $\nu=3 / 7$ state. 